\title{
Article \\ Gender Classification Using Proposed CNN-Based Model and Ant Colony Optimization
}

\author{
Farhat Abbas ${ }^{1}$, Mussarat Yasmin ${ }^{1}$, Muhammad Fayyaz ${ }^{1}$ (D), Mohamed Abd Elaziz ${ }^{2,3, *(\mathbb{D}), \text { Songfeng Lu }}{ }^{4,5, *(\mathbb{D})}$ \\ and Ahmed A. Abd El-Latif 6 (D)
}

1 Department of Computer Science, COMSATS University Islamabad, Wah Campus, WahCantt 47040, Pakistan; farhatabbas421@gmail.com (F.A.); mussaratabdullah@gmail.com (M.Y.); fayyazawan@ciitwah.edu.pk (M.F.) 2 Department of Mathematics, Faculty of Science, Zagazig University, Zagazig 44519, Egypt

3 Artificial Intelligence Research Center (AIRC), Ajman University, Ajman P.O. Box 346, United Arab Emirates

4 Hubei Engineering Research Center on Big Data Security, School of Cyber Science \& Engineering, Huazhong University of Science and Technology, Wuhan 430074, China

5 Research Institute of Huazhong University of Science and Technology in Shenzhen, Shenzhen 518057, China

6 Department of Mathematics and Computer Science, Faculty of Science, Menoufia University, Shebin El-Koom 32511, Egypt; aabdellatif@nu.edu.eg

* Correspondence: abd_el_aziz_m@yahoo.com (M.A.E.); lusongfeng@hust.edu.cn (S.L.)

check for updates

Citation: Abbas, F.; Yasmin, M.; Fayyaz, M.; Abd Elaziz, M.; Lu, S.; El-Latif, A.A.A. Gender Classification Using Proposed CNN-Based Model and Ant Colony Optimization. Mathematics 2021, 9, 2499. https:// doi.org/10.3390/math9192499

Academic Editor: Alma Y. Alanis

Received: 1 September 2021

Accepted: 30 September 2021

Published: 6 October 2021

Publisher's Note: MDPI stays neutral with regard to jurisdictional claims in published maps and institutional affiliations.

Copyright: (C) 2021 by the authors. Licensee MDPI, Basel, Switzerland. This article is an open access article distributed under the terms and conditions of the Creative Commons Attribution (CC BY) license (https:/ / creativecommons.org/licenses/by/ $4.0 /)$.

\begin{abstract}
Pedestrian gender classification is one of the key assignments of pedestrian study, and it finds practical applications in content-based image retrieval, population statistics, human-computer interaction, health care, multimedia retrieval systems, demographic collection, and visual surveillance. In this research work, gender classification was carried out using a deep learning approach. A new 64-layer architecture named 4-BSMAB derived from deep AlexNet is proposed. The proposed model was trained on CIFAR-100 dataset utilizing SoftMax classifier. Then, features were obtained from applied datasets with this pre-trained model. The obtained feature set was optimized with ant colony system (ACS) optimization technique. Various classifiers of SVM and KNN were used to perform gender classification utilizing the optimized feature set. Comprehensive experimentation was performed on gender classification datasets, and proposed model produced better results than the existing methods. The suggested model attained highest accuracy, i.e., $85.4 \%$, and 92\% AUC on MIT dataset, and best classification results, i.e., 93\% accuracy and 96\% AUC, on PKU-Reid dataset. The outcomes of extensive experiments carried out on existing standard pedestrian datasets demonstrate that the proposed framework outperformed existing pedestrian gender classification methods, and acceptable results prove the proposed model as a robust model.
\end{abstract}

Keywords: support vector machine; gender classification; visual surveillance; 4-BSMAB; ACS; CNN

\section{Introduction}

In recent years, researchers' interest in visual surveillance applications has been growing due to the availability of low-cost optical and infrared cameras and advanced computing machines. Digital cameras are widely used nowadays and deployed on roads, in shopping malls, metro lines and train stations, airports, and residential areas. With digital cameras, pedestrian images are captured under a specific field of view (FoV) in controlled environments [1]. These days, object recognition from images and videos captured by digital cameras is being preferred by people for automated tasks related to security monitoring, public safety [2], pedestrian behavior analysis, etc. Different approaches for video object detection based on deep learning were studied in [3]. Pattern classification from images was also carried out in [4-6]. Usually, the movement of different types of objects such as pedestrians takes place in images or video frames. Since pedestrians move in public areas for different purposes such as shopping, to go to work, or to go to school, they are a very important object of real life, and pedestrian-relevant tasks such as 
pedestrian face recognition [7], pedestrian tracking [8], pedestrian re-identification [9-11], action recognition [12,13], and pedestrian gender classification (PGC) [14] are becoming the focus of researchers. Since gender is a key attribute of a pedestrian and plays a role in social communication and human classification (male or female), gender prediction can be useful for various applications related to content-based image retrieval (CBIR), population statistics, human-computer interaction (HCI), health care, multimedia retrieval systems, demographic collection [15], and visual surveillance. Keeping in view the importance of gender prediction in these application areas, pedestrian gender analysis is becoming an imperative field of computer vision. In recent decades, considerable progress has been made in performing the task of gender recognition. Some of the introduced automated gender recognition methods use only low-level information and some others utilize highlevel information of images. Low-level information includes hand-crafted features such as shape, color, and texture, while high-level information includes deep features of images [16-20]. These information types also usually use pedestrians' voices, gait, skin color, and facial expression for gender prediction [21]. However, these approaches have faced issues related to different camera settings, pedestrians' complex full-body appearances, and variations in their poses. Moreover, environmental effects which include changes in brightness, viewpoint disparities, blur, occlusion, and background cluttering, and images having a low resolution have also affected results while classifying pedestrian gender. Hence, designing a robust method which can effectively automate the process of gender prediction is required. To address the above-mentioned issues of gender prediction, previously proposed methods follow a two-stage classification framework that first extracts features and then carries out classification. Commonly, during the first stage, designing a highly representative feature descriptor is considered for gender, and then a precise binary classifier distinguishing male and female is obtained during the second stage. Naturally, feature representation is required to be discriminative as well as robust. For this, several methods have used facial characteristics using complete faces [22-25], and various hand-crafted features have been developed utilizing facial images such as histograms of gradients (HOGs) [26], iris codes [27], and local binary patterns (LBPs) [28], for gender recognition. In automated surveillance systems, since poor visible pedestrian face images are captured due to the distance between the camera and pedestrian faces, especially in long-distance conditions, relevant methods produce low gender recognition rates and are not effective for image-based pedestrian gender recognition. Similarly, the approaches which also use face images do not work in scenarios where a camera is used to capture images of pedestrians from their different sides such as back, left, or right side. The reason behind this is that pedestrian images captured in multi-camera environments normally have issues such as scene variations, viewing angle variation, occlusions, blur, and changes in brightness, and due to these issues, it becomes difficult to extract the required face information. Scene variations lead to changes in the background and illumination of an image, while viewpoint variations cause changes in pedestrians' full-body appearances and postures. Among these issues, viewpoint variation is considered critical because of its impact on diversification in pedestrian full-body appearances and postures, hence making pedestrian gender recognition tasks more difficult. Deep neural network (DNN)-based methods, especially convolutional neural network (CNN)-based classifiers, performed more successfully than hand-crated features in various tasks related to computer vision, as $\mathrm{CNN}$ learns and generates effective feature representations from input data under the diverse appearances of gender and various camera settings [29,30]. Some other methods have tackled scene variation issues [31-34], viewpoint variation concerns [35-38], both scene and viewpoint variation issues [39], and combinations of aforementioned problems [40,41] for pedestrian gender recognition. However, these methods normally need large-scale datasets for training when learning effective models. Even though both traditional and deep CNN approaches have generated effective benchmark results while performing gender prediction, they are still lacking in terms of challenging issues such as distinct feature representations, an imbalanced distribution of data, lower accuracies, and a small sample 
space (SSS) for model learning. Regarding these scenarios, the objective of this research work is to develop a new method for pedestrian gender recognition by tackling viewpoint variation concerns for better performance.

In this work, the proposed solution for gender classification provides a robust categorization of full-body view-based pedestrian images. The proposed method is related to offline PGC which utilizes body clues in pedestrian image classification. Four different views such as front, back, side, and mixed are considered for PGC. In this regard, a 64 layer-based CNN model is presented to obtain and learn features. The learned features are then supplied to the optimization approach. The features obtained from this approach are provided to different classifiers for PGC. Keeping in view the fact of non-availability of large datasets of PGC for the training of proposed network, to overcome this issue, a large dataset, i.e., CIFAR-100, was selected to train the proposed model, after which feature sets of testing dataset were extracted from a fully connected layer of proposed CNN-based model. Extensive experimentation was carried out using several variations of optimized feature subsets. From the results obtained, it is observed that an overall accuracy of $85.4 \%$ and $92 \%$ AUC is achieved on MIT dataset with Fine KNN variant of KNN classifier, and best classification accuracy of $93 \%$ is attained on PKU-Reid dataset with Cubic SVM classifier, and selection of a 1000-feature subset. The major contributions are presented below:

- A new architecture based on 64 layers named 4-BSMAB is proposed to obtain features from images. Due to the non-availability of larger datasets, the training of proposed model is carried out on CIFAR-100 dataset, and then the trained model is utilized to extract features from the testing datasets.

- The feature optimization approach (ACS) is applied to obtain features for dimension reduction of extracted features.

- Various classifiers are tested for PGC, and then the most successful classifier is benchmarked. The classification accuracy achieved with the proposed model shows that the proposed framework is acceptable.

The remaining sections of this manuscript are organized as follows: The Introduction section describes the proposed domain, and next section explains literature review. Section 3 describes the proposed framework. The fourth section provides the results and discusses the details. At the end of this manuscript, this research work is finally concluded.

\section{Related Work}

In this section, a summary of relevant existing techniques used for gender classification is presented. The following approaches have been proposed for view-based PGC in the relevant literature.

\subsection{Traditional/Hand-Crafted Feature-Based Approaches}

In this section, a summary of methods that use hand-crafted features for gender classification is highlighted. These approaches use low-level information (features related to shape, color, texture, etc.). For instance, Cao et al. [42] proposed an algorithm named partbased gender recognition (PBGR) utilizing fixed frontal or back views of gender full-body appearance to obtain edge map-based shape information, HOGs, and raw information. They achieved $76.0 \%, 74.6 \%$, and $75.0 \%$ accuracy on front views, back views, and non-fixed views, respectively. Furthermore, Guo et al. [43] utilized front views, back views, and mixed views to investigate biologically inspired features (BIF) from the human body to handle pose variations with support vector machine (SVM). For manifold learning, unsupervised principal component analysis (PCA), supervised orthogonal locality preserving projections (OLPP), marginal Fisher analysis (MFA), and locality-sensitive discriminant analysis (LSDA) were utilized. They achieved $79.5 \%, 84.0 \%$, and $79.2 \%$ accuracy on frontal view with BIF+LSDA, back view with BIF+LSDA, and mixed views with BIF+PCA, respectively, on MIT dataset. Collins et al. [44] extracted features related to spatial pyramid HOGs (PHOGs), local HSV (LHSV) color histograms, spatial pyramid bag of words, etc., and 
used mixed views from static full-body images to investigate image representations. They obtained $72.2 \%, 76.0 \%$, and $80.6 \%$ overall accuracy on uncropped MIT, cropped MIT, and uncropped VIPeR dataset images, respectively. In addition to above, Geelen et al. [45] first obtained hand-crafted features such as shape, color, and texture from full-body view-based images. Then, a combination of these features was used to perform experiments on MIT CBCL dataset and Datasets A and B for gender classification using SVM and random forest (RF) kernel. They obtained $81.6 \%, 82.7 \%$, and $80.9 \%$ overall accuracy on front views, back views, and mixed views, respectively. They also achieved $79.0 \%, 79.3 \%$, and $76.6 \%$ mean accuracy on front views, back views, and mixed views on MIT dataset. With the above gender classification techniques, although it has been observed that hand-crafted features (low-level feature representations) provide significant resistance against illumination and pose issues, obtaining distinct features from pedestrian full-body views with complex appearances is another challenging issue. Therefore, further investigation of pedestrian full-body views is required to obtain more definite and optimum information for gender classification.

\subsection{Deep Learning-Based Approaches}

To cope with the problems raised by the traditional hand-crafted feature-based gender classification techniques discussed above such as pedestrians' diverse appearances and captured images having a low resolution, deep CNN models have been proposed and are considered more appropriate [46,47]. The CNN architecture is popular because of its significant advances in the accuracy obtained in different classification studies [48-50]. Currently, trained deep CNN models have been used in a few existing methods for gender prediction. For instance, $\mathrm{Ng}$ et al. [16] utilized a CNN model comprising seven layers for issues related to the domain of gender classification. The training of CNN model was carried out on MIT pedestrian dataset for the prediction of gender classification. Overall accuracies of $80.4 \%$ and $79.2 \%$ were obtained on both front and rear views with a view classifier and without a view classifier, respectively. The proposed approach performed successfully on homogeneous datasets of a small size. Antipov et al. [17] applied mini$\mathrm{CNN}$ and AlexNet-CNN to learn features and compare them with hand-crafted features (HOG) to solve the issue of image feature selection. They found MAP values of 0.80 and 0.85 and AUC values of 0.88 and 0.91 on familiar datasets, while they found MAP values of 0.75 and 0.79 and AUC values of 0.80 and 0.85 on unfamiliar datasets using mini-CNN and AlexNet-CNN. The results showed that the learned features significantly outperformed hand-crafted features for heterogeneous datasets. $\mathrm{Ng}$ et al. [18] utilized grayscale, RGB, and YUV color spaces on pedestrians' full-body images to represent the image for gender prediction with a deep CNN network, which produced significant results on MIT dataset containing pedestrians' front and rear views. An average accuracy of $81.47 \%$ was attained on frontal and rear views with the grayscale color space. $\mathrm{Ng}$ et al. [19] further utilized labeled low-level training data and a CNN to introduce a strategy for training. Filters were learned with k-means clustering (unsupervised learning), whereas supervised learning performed pre-training on MIT dataset (front and back views). The training strategy generally performed better than random weight initialization. Raza et al. [20] used appearances from the complete and upper portion of body and deep CNN model for the analysis of pedestrian gender. The existing mechanism for pedestrian parsing was applied to parse both full-body as well as upper-half body-based pedestrian objects in CNN model, after which the SoftMax classifier was applied. The authors achieved $82.1 \%, 81.3 \%$, and $82.0 \%$ overall accuracy and $81.1 \%, 81.7 \%$, and $80.7 \%$ mean accuracy on front views, back views, and mixed views with full-body appearances. They also obtained $83.3 \%, 82.3 \%$, and $82.8 \%$ overall accuracy and $80.5 \%, 82.3 \%$, and $81.4 \%$ mean accuracy on front views, back views, and mixed views with upper-body appearances. Furthermore, Raza et al. [51] also used a deep learning approach and a stacked sparse autoencoder (SSAE) to classify gender. The deep neural network method parsed pedestrian images to remove background, and a two-layer SSAE with SoftMax classifier predicted 
gender as male or female. The researchers achieved $82.9 \%, 81.8 \%$, and $82.4 \%$ accuracy on front views, back views, and mixed views, respectively, on MIT dataset. They also attained a 91.5\% AUC mean value on PETA dataset. Cai et al. [52] investigated and obtained deep features and low-level (HOG) features simultaneously from images by a deep CNN model named as deep-learned and hand-crafted features fusion network (DHFFN) using PCA. After extracting features, fusion is applied to mix both features for exploring their full merits. Experiments on numerous public datasets such as MIT, VIPeR, GRID, PRID, and CHUK were performed, and DHFFN produced 0.95 MAP and 0.95 AUC and was declared a better performer than the state-of-the-art gender prediction methods. Cai et al. [40] further introduced HOG-assisted deep feature learning (HDFL), a novel method that uses a deep CNN to cater common challenges such as viewpoint variations, occlusion, and poor quality faced while predicting gender. HDFL efficiently extracted deep-learned features as well as HOG features simultaneously from the pedestrian picture. A feature fusion process is then applied to extract more discriminative features to provide to SoftMax classifier for gender prediction. The proposed HDFL achieved 0.93 MAP and 0.94 AUC with local response normalization (LRN) and 0.94 MAP and 0.95 AUC with LRN. In earlier gender classification studies, CNN architecture has been used only for considering whole-body images, i.e., global information, but $\mathrm{Ng}$. et al. [53] took global as well as local information from full-body images and introduced a novel parts-based framework that uses a combination of local and global information towards PGC. A local and global CNN method was trained on both whole-body images as well as identified areas of body for feature learning and classification. While comparing the accuracy extracted by utilizing different regions of body such as upper, middle, and lower regions after performing experiments on MIT and APiS datasets, the upper-half body region played a more important role in gender classification as compared to the middle or lower half of body. The authors achieved $84.4 \%, 88.9 \%$, and $86.8 \%$ accuracy utilizing a combination of MIT and APis datasets on frontal, non-frontal, and mixed views, respectively. Fayyaz et al. [41] proposed a hybrid approach that produces a combination of low-level information and deep features of pedestrian images and computes information effectively assisted bya joint feature representation (JFR) scheme for better gender classification tasks. Extensive experiments were performed by adopting different classifiers such as SVM, discriminant classification, and k-nearest neighbor (KNN) to observe sufficient low-level (HOG and LOMO features) and deep feature-based contributions for the design of a JFR, and in this way, the proposed approach achieved 96\% AUC and 89.3\% accuracy on PETA dataset, and $86 \%$ AUC and $82 \%$ accuracy on MIT dataset. A study was also conducted by Cai et al. [39] in which the cascading scene and viewpoint feature learning (CSVFL) method improved pedestrian gender recognition. In CSVFL, two crucial challenges, namely, scene and viewpoint variations in pedestrian gender recognition, were jointly considered. The authors demonstrated that CSVFL was able to resist both variations (scene and viewpoint) at the same time. The results generated by CSVFL were also compared with various recent relevant tasks, and an excellent performance was observed. They obtained $84.4 \%, 85.9 \%$, and $85.2 \%$ accuracy utilizing MIT dataset on frontal, back, and mixed views, respectively. They also achieved $81.9 \%, 84.7 \%, 72.1 \%$, and $80.1 \%$ accuracy utilizing VIPeR dataset on frontal, back, side, and mixed views. Further, $92.4 \%, 94.6 \%, 88.2 \%$, and $92.7 \%$ accuracy was obtained using PETA dataset on frontal, back, side, and mixed views, respectively.

It can be observed from the results of above-mentioned fine-tuned models that these models are robust, but imbalanced distribution of data is also a challenge while obtaining class-wise accuracy. The above discussion also reflects the fact that full-body view-based pedestrian images are widely investigated for gender classification. All the existing techniques equally applied large-scale and small-scale datasets in experimentation. It has been observed that a fusion approach generates a compact representation of gender images for classification. Moreover, a dataset having a small size is an issue for model learning in deep learning-based approaches. 


\section{Material and Methods}

This section presents the proposed model 4-BSMAB and its major steps for PGC. These steps include pre-training of proposed model, dataset balancing, process of feature extraction from 4-BSMAB model, ACS-based feature optimization, and, at the end, classification. An overview of this model is presented in Figure 1. These steps are elaborated in the upcoming section.

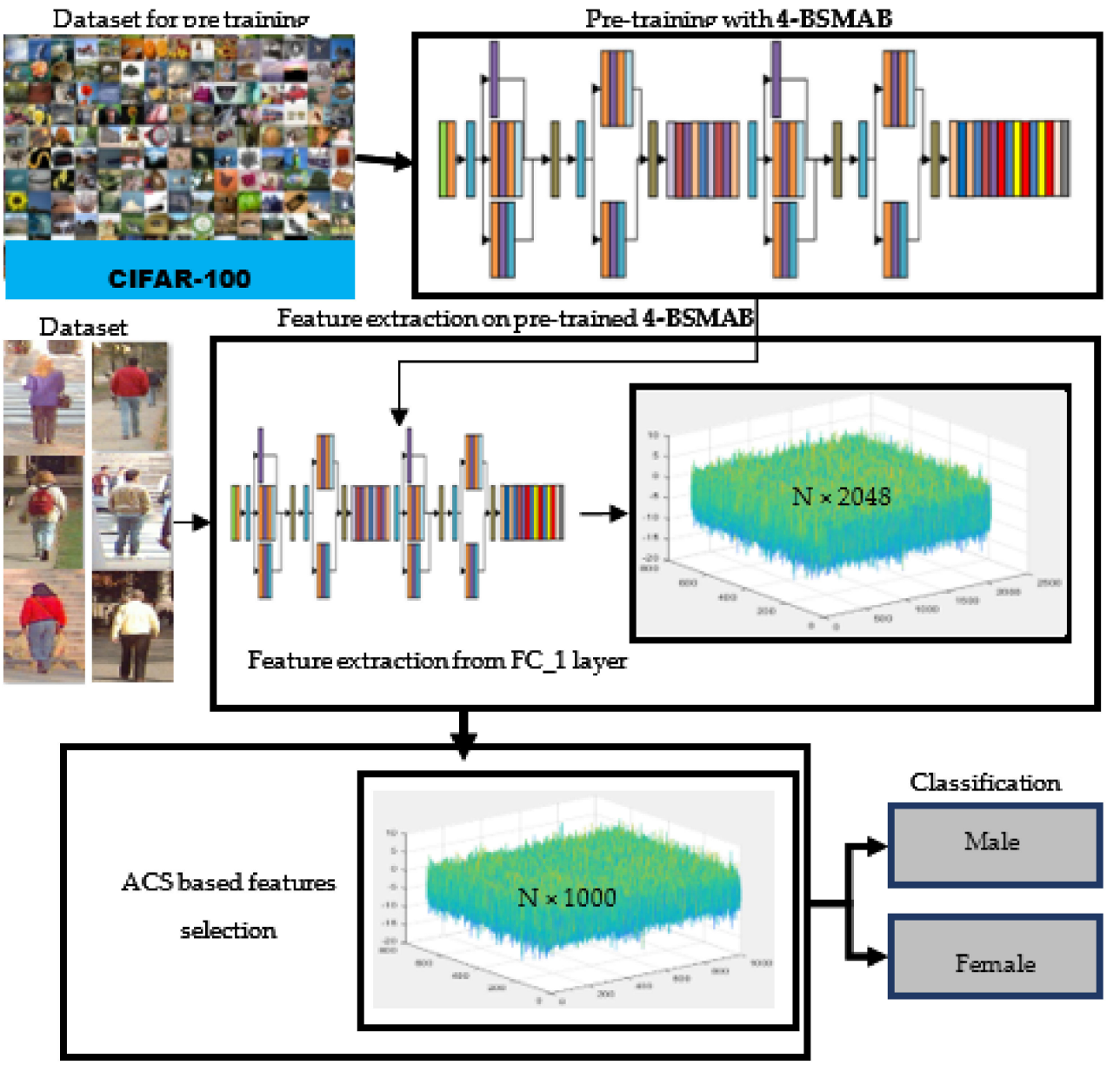

Figure 1. Proposed 4-BSMAB model for pedestrian gender classification.

\subsection{4-BSMAB}

A new architecture, 4-BSMAB (4-branch subnets with modified AlexNet backbone), based on CNN architecture is introduced in this work for PGC. This newly developed model is derived from CNN network named AlexNet [54]. AlexNet contains 25 layers including 5 convolutional layers, 3 fully connected layers, 3 pooling layers, 7 rectified linear unit (ReLU) layers, 2 dropout layers, and SoftMax layers and is divided into 3 repeating blocks named here as R1, R2, and R3. The new model contains 64 layers including input and output layers. The architectural view of proposed model 4-BSMAB is presented in Figure 2, and the details of layers are listed in Table 1. 
Table 1. Configurations of layers of proposed model 4-BSMAB.

\begin{tabular}{|c|c|c|c|c|c|c|}
\hline Layer \# & $\begin{array}{l}\text { Layer } \\
\text { Name }\end{array}$ & Feature Maps & Filter Depth & Stride & Padding & $\begin{array}{l}\text { Pooling } \\
\text { Window } \\
\text { Size/Other } \\
\text { Values }\end{array}$ \\
\hline 1 & Data & $227 \times 227 \times 3$ & & & & \\
\hline 2 & C_1 & $55 \times 55 \times 96$ & $11 \times 11 \times 3 \times 96$ & [4 4] & {$\left[\begin{array}{llll}0 & 0 & 0 & 0\end{array}\right]$} & \\
\hline 3 & R1_1 & $55 \times 55 \times 96$ & & & & \\
\hline 4 & C4_1 & $55 \times 55 \times 96$ & $5 \times 5 \times 96 \times 96$ & {$\left[\begin{array}{ll}1 & 1\end{array}\right]$} & Same & \\
\hline 5 & $\mathrm{C} 2 \_1$ & $55 \times 55 \times 48$ & $1 \times 1 \times 96 \times 48$ & {$\left[\begin{array}{ll}1 & 1\end{array}\right]$} & Same & \\
\hline 6 & BN1_1 & $55 \times 55 \times 48$ & & & & \\
\hline 7 & BN2 & $55 \times 55 \times 96$ & & & & \\
\hline 8 & BN3_1 & $55 \times 55 \times 96$ & & & & \\
\hline 9 & LR2_1 & $55 \times 55 \times 96$ & & & & $\begin{array}{c}\text { Scaling } \\
\text { value } 0.01\end{array}$ \\
\hline 10 & C3_1 & $55 \times 55 \times 96$ & $11 \times 11 \times 48 \times 96$ & {$\left[\begin{array}{ll}1 & 1\end{array}\right]$} & Same & \\
\hline 11 & LR1_1 & $55 \times 55 \times 96$ & & & & $\begin{array}{c}\text { Scaling } \\
\text { value } 0.01\end{array}$ \\
\hline 12 & ADD1_1 & $55 \times 55 \times 96$ & & & & \\
\hline 13 & R1_2 & $55 \times 55 \times 96$ & & & & \\
\hline 14 & $\mathrm{C} 4 \_2$ & $55 \times 55 \times 96$ & $5 \times 5 \times 96 \times 96$ & {$\left[\begin{array}{ll}1 & 1\end{array}\right]$} & Same & \\
\hline 15 & BN3_2 & $55 \times 55 \times 96$ & & & & \\
\hline 16 & LR2_2 & $55 \times 55 \times 96$ & & & & $\begin{array}{c}\text { Scaling } \\
\text { value } 0.01\end{array}$ \\
\hline 17 & C2_2 & $55 \times 55 \times 48$ & $1 \times 1 \times 96 \times 48$ & {$\left[\begin{array}{ll}1 & 1\end{array}\right]$} & Same & \\
\hline 18 & BN1_2 & $55 \times 55 \times 48$ & & & & \\
\hline 19 & C3_2 & $55 \times 55 \times 96$ & $11 \times 11 \times 48 \times 96$ & {$\left[\begin{array}{ll}1 & 1\end{array}\right]$} & Same & \\
\hline 20 & LR1_2 & $55 \times 55 \times 96$ & & & & $\begin{array}{c}\text { Scaling } \\
\text { value } 0.01\end{array}$ \\
\hline 21 & ADD1_2 & $55 \times 55 \times 96$ & & & & \\
\hline 22 & norm1 & $55 \times 55 \times 96$ & & & & \\
\hline 23 & P1 & $27 \times 27 \times 96$ & & [2 2] & {$\left[\begin{array}{llll}0 & 0 & 0 & 0\end{array}\right]$} & $\begin{array}{c}\text { Maximum } \\
\text { pooling } \\
3 \times 3\end{array}$ \\
\hline 24 & $\mathrm{BN} 4$ & $27 \times 27 \times 96$ & & & & \\
\hline 25 & GC1(c5) & $27 \times 27 \times 256$ & $\begin{array}{c}\text { Two groups of } \\
5 \times 5 \times 48 \times 128\end{array}$ & {$\left[\begin{array}{ll}1 & 1\end{array}\right]$} & {$\left[\begin{array}{llll}2 & 2 & 2 & 2\end{array}\right]$} & \\
\hline 26 & $\mathrm{R} 2$ & $27 \times 27 \times 256$ & & & & \\
\hline 27 & norm2 & $27 \times 27 \times 256$ & & & & \\
\hline 28 & P2 & $13 \times 13 \times 256$ & & [2 2 2] & {$\left[\begin{array}{llll}0 & 0 & 0 & 0\end{array}\right]$} & $\begin{array}{c}\text { Maximum } \\
\text { pooling } \\
3 \times 3\end{array}$ \\
\hline 29 & BN5 & $13 \times 13 \times 256$ & & & & \\
\hline 30 & GC2(c6) & $13 \times 13 \times 384$ & $3 \times 3 \times 256 \times 384$ & {$\left[\begin{array}{ll}1 & 1\end{array}\right]$} & {$\left[\begin{array}{llll}1 & 1 & 1 & 1\end{array}\right]$} & \\
\hline 31 & R3_1 & $13 \times 13 \times 384$ & & & & \\
\hline 32 & BN7 & $13 \times 13 \times 384$ & & & & \\
\hline 33 & C7_1 & $13 \times 13 \times 192$ & $1 \times 1 \times 384 \times 192$ & {$\left[\begin{array}{ll}1 & 1\end{array}\right]$} & Same & \\
\hline
\end{tabular}


Table 1. Cont.

\begin{tabular}{|c|c|c|c|c|c|c|}
\hline Layer \# & $\begin{array}{l}\text { Layer } \\
\text { Name }\end{array}$ & Feature Maps & Filter Depth & Stride & Padding & $\begin{array}{c}\text { Pooling } \\
\text { Window } \\
\text { Size/Other } \\
\text { Values }\end{array}$ \\
\hline 34 & BN6_1 & $13 \times 13 \times 192$ & & & & \\
\hline 35 & C8_1 & $13 \times 13 \times 384$ & $5 \times 5 \times 192 \times 384$ & {$\left[\begin{array}{ll}1 & 1\end{array}\right]$} & Same & \\
\hline 36 & LR3_1 & $13 \times 13 \times 384$ & & & & $\begin{array}{c}\text { Scaling } \\
\text { value } 0.01\end{array}$ \\
\hline 37 & C9_1 & $13 \times 13 \times 384$ & $3 \times 3 \times 384 \times 384$ & {$\left[\begin{array}{ll}1 & 1\end{array}\right]$} & Same & \\
\hline 38 & BN8_1 & $13 \times 13 \times 384$ & & & & \\
\hline 39 & LR4_1 & $13 \times 13 \times 384$ & & & & $\begin{array}{c}\text { Scaling } \\
\text { value } 0.01\end{array}$ \\
\hline 40 & ADD2_1 & $13 \times 13 \times 384$ & & & & \\
\hline 41 & R3_2 & $13 \times 13 \times 384$ & & & & \\
\hline 42 & C7_2 & $13 \times 13 \times 384$ & $1 \times 1 \times 384 \times 192$ & {$\left[\begin{array}{ll}1 & 1\end{array}\right]$} & Same & \\
\hline 43 & C9_2 & $13 \times 13 \times 384$ & $3 \times 3 \times 384 \times 384$ & {$\left[\begin{array}{ll}1 & 1\end{array}\right]$} & Same & \\
\hline 44 & BN8_2 & $13 \times 13 \times 384$ & & & & \\
\hline 45 & BN6_2 & $13 \times 13 \times 192$ & & & & \\
\hline 46 & C8_2 & $13 \times 13 \times 384$ & $5 \times 5 \times 192 \times 384$ & {$\left[\begin{array}{ll}1 & 1\end{array}\right]$} & Same & \\
\hline 47 & LR3_2 & $13 \times 13 \times 384$ & & & & $\begin{array}{c}\text { Scaling } \\
\text { value } 0.01\end{array}$ \\
\hline 48 & LR4_2 & $13 \times 13 \times 384$ & & & & $\begin{array}{c}\text { Scaling } \\
\text { value } 0.01\end{array}$ \\
\hline 49 & ADD2_2 & $13 \times 13 \times 384$ & & & & \\
\hline 50 & GC3(c10) & $13 \times 13 \times 384$ & $\begin{array}{c}\text { Two groups of } \\
3 \times 3 \times 192 \times 192\end{array}$ & {$\left[\begin{array}{ll}1 & 1\end{array}\right]$} & {$\left[\begin{array}{llll}1 & 1 & 1 & 1\end{array}\right]$} & \\
\hline 51 & $\mathrm{R} 4$ & $13 \times 13 \times 256$ & & & & \\
\hline 52 & GC4(c11) & $13 \times 13 \times 256$ & $\begin{array}{c}\text { Two groups of } \\
3 \times 3 \times 192 \times 128\end{array}$ & {$\left[\begin{array}{ll}1 & 1\end{array}\right]$} & {$\left[\begin{array}{llll}1 & 1 & 1 & 1\end{array}\right]$} & \\
\hline 53 & R5 & $13 \times 13 \times 256$ & & & & \\
\hline 54 & P3 & $6 \times 6 \times 256$ & & [2 2] & {$\left[\begin{array}{llll}0 & 0 & 0 & 0\end{array}\right]$} & $\begin{array}{c}\text { Max } \\
\text { pooling } \\
3 \times 3\end{array}$ \\
\hline 55 & BN9 & $6 \times 6 \times 256$ & & & & \\
\hline 56 & Fc_1 & $1 \times 1 \times 2048$ & & & & \\
\hline 57 & R6 & $1 \times 1 \times 2048$ & & & & \\
\hline 58 & D1 & $1 \times 1 \times 2048$ & & & & $\begin{array}{c}50 \% \\
\text { Dropout }\end{array}$ \\
\hline 59 & Fc_2 & $1 \times 1 \times 2048$ & & & & \\
\hline 60 & R7 & $1 \times 1 \times 2048$ & & & & \\
\hline 61 & D2 & $1 \times 1 \times 2048$ & & & & $\begin{array}{c}50 \% \\
\text { Dropout }\end{array}$ \\
\hline 62 & Fc_3 & $1 \times 1 \times 100$ & & & & \\
\hline 63 & prob & $1 \times 1 \times 100$ & & & & \\
\hline 63 & $\begin{array}{l}\text { Class } \\
\text { output }\end{array}$ & & & & & \\
\hline
\end{tabular}


The existing network, i.e., AlexNet, was altered with the help of addition of layers, and a new model, 4-BSMAB, is proposed. The batch normalization (BN) layer is included at the end of blocks R1 and R2. Two branched sub-networks are also added along with $\mathrm{R}$ blocks. BN1_1 is called first sub-network, and it has three branches. The first branch has a BN tier. The second branch has C2, BN1, C3, and LR1 tiers. The last branch contains layers such as C9, BN8, and LR4. Then, the fusion process is applied on three branches with an addition (ADD) layer. The other sub-network (BN_2) has only two branches. The difference between BN_2 and BN_1 is that BN_1 has only the group BN layer. Two sub-networks such as BN_1 and BN_3 are incorporated at the end of ReLU layer in block R1. Two activation functions are used, i.e., ReLU and Leaky ReLU, at ReLU layer. The ReLU functions are simple and fast and help in speeding up the training phase, and this way, neural networks are also improved. The ReLU functions are easy to compute and do not suffer from vanishing gradients. These functions are implemented with a simple procedure, and due to this characteristic, they are suitable to be used on GPUs.

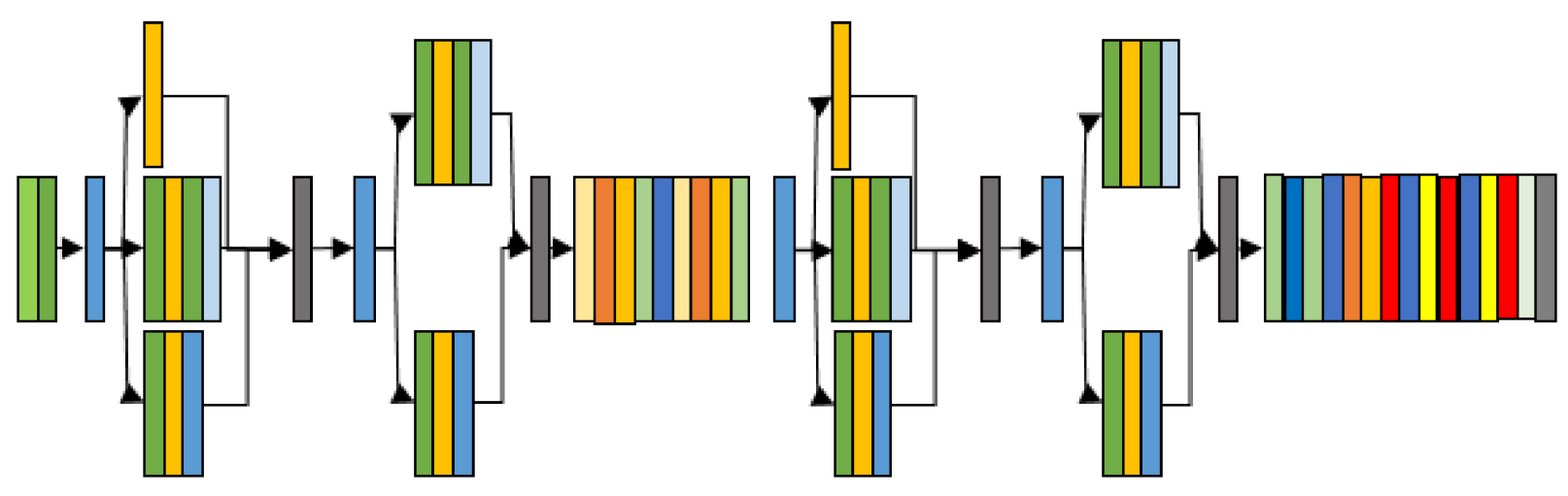

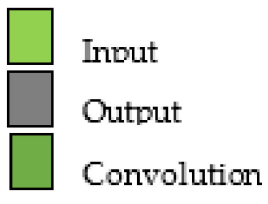

Convolution
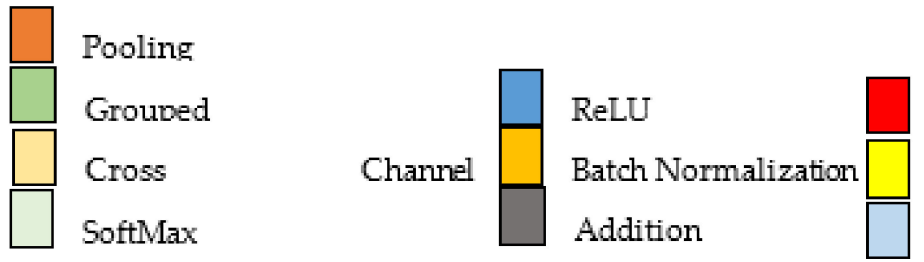

Fullv Connected

Dropout

Leaky ReLU

Figure 2. Architectural view of proposed model 4-BSMAB.

GPUs are popular and can be improved while carrying out matrix operations. Leaky ReLU functions stop ReLU problems from dying. This type of variation of ReLU produces a small positive slope in the negative area; therefore, it produces possible back-propagation, even for negative input values. Leaky ReLU does not provide steady predictions for input values which are negative. Two sub-networks including BN6_1 and BN8_1 are incorporated at the end of block R2.

The layer details of proposed 4-BSMAB are discussed in the upcoming section. The convolution of input vector $\|_{j-1}$ is carried out using a filter bank in C_1 layer. The mathematical form of convolution operation represented by $*$ is described as

$$
\|_{p^{\prime}, j}=\mathcal{N}_{j}\left(\sum_{p} \mathfrak{H}_{j, p^{\prime} p} * \|_{p, j-1}+\mathcal{M}_{p^{\prime}, j}\right)
$$

where $p_{j}$ represents various input channels, and $p_{j}^{\prime}$ represents the number of output channels; the value represented by $\mathrm{j}$ indicates the number of layers [55]; $\mathfrak{H}$ denotes the filter of depth $p^{\prime}{ }_{j}$; and both symbols $\mathcal{M}$ and $\mathcal{N}$ indicate nonlinear functions. Layers such as group convolution (GC) are also added in 4-BSMAB model. A GC layer is a combination of many convolutional layers. A GC layer enables the procedure of training around GPUs which are in the form of clusters and have a low capacity related to memory. The filters are 
divided into many splits in a GC layer. A certain range of collection of 2D convolution is carried out by all groups. The mathematical form of layers related to pooling is

$$
\left\|_{i, j, x, v}=\max _{l=1 \ldots s, m=1 \ldots . .}\right\|_{p, j-1,(\mathrm{w}+\mathrm{m})(\mathrm{x}+\mathrm{n})}
$$

where $w, x$ represent the index of matrix of image $\mathbb{I}_{p, j-1}$, and $m, n$ denote the index of matrix used for the selected pool window. Both norm(s) and $\mathrm{BN}(\mathrm{s})$ are utilized in this scheme. $\mathrm{BN}$ [56] is a procedure to adjust neurons of the channel over the amount defined for a small batch. Its purpose is to determine both the mean and variance in parts. With the help of a determined mean, the separation of features is carried out with standard deviation. The mean of batch $\mathcal{B}=\mathbb{I}_{1}, \ldots, \mathbb{I}_{w}$ is calculated as follows:

$$
\operatorname{Mean}_{\mathcal{B}}=\frac{1}{w} \sum_{z=1}^{w} \mathbb{I}_{z}
$$

where $w$ shows per batch feature maps. The variance is described per batch (small) and is shown as

$$
\operatorname{Var}_{\mathbb{B}}=\frac{1}{w} \sum_{z=1}^{w}\left(\mathbb{I}_{z}-\operatorname{Mean}_{\mathbb{B}}\right)^{2}
$$

The below expression is then used forfeature normalization.

$$
\hat{\mathbb{I}_{Z}}=\frac{\mathbb{I}_{z}-\operatorname{Mean}_{\mathbb{B}}}{\sqrt{\operatorname{Var}_{\mathbb{B}}+\mathcal{D}}}
$$

where $\mathcal{D}$ represents the consistency value, but it remains constant. The norm layer is used for simplification. The norm layer involves scaling pixels with the maximum factor for local prior layers and boosts the spatial-visual quality. The norm equation is

$$
\| \overline{\overline{\mathbb{I}_{Z}}}=\frac{\mathbb{I}_{z}}{\left(o+\frac{\beth * \Im}{N}\right)^{\wp}}
$$

where $\overline{\overline{\mathbb{I}_{Z}}}$ represents the obtained feature map at the end of norm, $\Im$ shows the "sum of square", $N$ denotes the size of channel, and $o, \beth$, and $\wp$ represent normalization criteria. The 4-BSMAB model utilizes both $R$ and LR. The standard $R$ transforms numbers less than zero to zero and is shown in [57] as

$$
\|_{u, \ddot{\mathrm{r}}}=\operatorname{maximum}\left(0, \|_{u, \ddot{\mathrm{r}}}\right)
$$

For values below 0 , LR has a small slope instead of becoming 0 . LR would have $\ddot{r}=0.01 \ddot{u}$ when $\ddot{u}$ is negative. Some other works [58-60] are also available from which CNN in-depth learning is possible.

\subsection{Pre-Training of Proposed Model and Feature Extraction}

The proposed model 4-BSMAB extracts features from the pipeline deeply trained by CNN. The training of proposed model is carried out on a dataset named CIFAR100 [61] having 100 classes of images. In this repository, each class of images is divided into 500 images for model learning and 100 images for model validation. For pre-training, learning images, as well as validation images, are mixed such that each class contains 600 images. The resultant dataset after mixing both types of images of each class is provided to the proposed CNN model for training purposes. The finally trained network is then applied to extract features on pedestrian attribute recognition datasets [41], and FC_1 layer is selected to obtain features. A total of 2048 features are extracted from each image from this layer. This produces a feature set having $418 \times 2048,470 \times 2048$, and $1728 \times 2048$ dimensions for frontal views, back views, and mixed views, respectively, for MIT dataset. This also produces a feature set with $590 \times 2048,331 \times 2048$, and $1264 \times 2048$ dimensions for frontal 
views, back views, and mixed views for VIPeR dataset, and feature set dimensions of $684 \times 2048,228 \times 2048$, and $1640 \times 2048$ for frontal views, back views, and mixed views for PKU-Reid dataset. Some intermediate visualizations of features captured at various stages of convolution performed by the proposed model 4-BSMAB are shown in Figure 3.

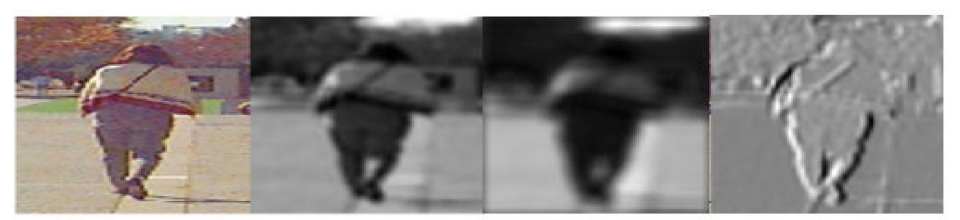

(a)

(b)

(c)

(d)

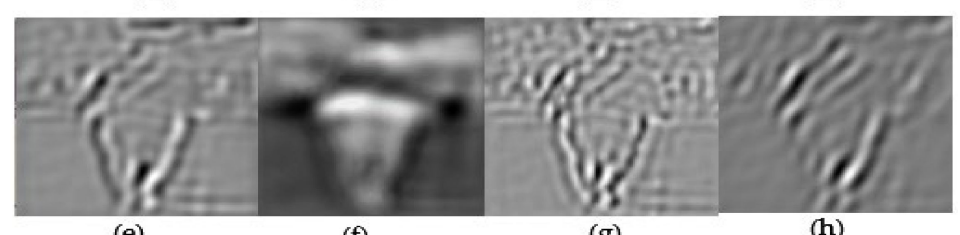

(e)

(f)

(g)

(h)

Figure 3. 4-BSMAB best activation visualizations of an image at various convolutional layers: (a) original image; (b) C1_1; (c) C2_1; (d) C2_2; (e) C3_1; (f) C3_2; (g) C4_2; (h) C7_1.

\subsection{Feature Selection Based on ACS Optimization}

The entropy operation [62] is used to code the obtained features. Entropy e is usually applied to score the features. Mathematically, entropy has the following form:

$$
\ddot{\mathrm{e}}\left(\left\|_{1}^{\prime}, \ldots,\right\|_{n}^{\prime}\right)=-\sum_{\mathfrak{f}_{1}} \ldots \sum_{\mathfrak{f}_{n}} P\left(\mathfrak{f}_{1}, \ldots, \mathfrak{f}_{n}\right) \operatorname{LOG} P\left(\mathfrak{f}_{1}, \ldots, \mathfrak{f}_{n}\right)
$$

where $\left(\left\|_{1}^{\prime}, \ldots,\right\|_{n}^{\prime}\right)$ show the features, $\left(\mathfrak{f}_{1}, \ldots, \mathfrak{f}_{n}\right)$ represent random variables, and $P\left(\mathfrak{f}_{1}, \ldots, \mathfrak{f}_{n}\right)$ calculate the probability. ACS is a learning-based approach used for feature optimization. When it is combined with entropy-based feature selection, it becomes an embedded approach. The obtained entropy-coded scores are provided to ACS for feature optimization. The ACS is related to ants' activities and their movements [63]. Ants move between places and diffuse material called "pheromones". With time, the material strength decreases gradually. The ants follow the way while calculating the probability of pheromones. This helps ants to select the least expensive path. Therefore, ants' movement between places is similar to the movement that takes place between vertices of a graph. A graph vertex indicates a feature, and edges from a vertex to another vertex show the selection of features. The strategy repeats to find the best features. The approach stops when minimum number of vertices is traversed and a set criterion is satisfied. The linking arrangement of vertices is similar to a mesh. An ant selects features on a probability basis at a given point at any specified time, and this is written as

$$
P_{j}^{m}(\mathrm{~T})=\left\{\begin{array}{c}
\frac{\left|h_{j}(\mathfrak{t})\right|^{w}\left|E_{j}\right|^{w}}{\sum_{\left.v \in \mathbf{e}_{\left(\mathbb{I}^{\prime}\right.}, \ldots, \mathbb{I}_{n}^{\prime}\right)}\left|\mathfrak{h}_{v}(\mathcal{T})\right|^{\mathfrak{w}}\left|\mathfrak{S}_{v}\right|^{\mathfrak{w}}} \text { if } j \in \ddot{\mathrm{e}}\left(\left\|_{1}^{\prime}, \ldots,\right\|_{n}^{\prime}\right) \\
0 \text { otherwise }
\end{array}\right.
$$

where $\ddot{\mathrm{e}}\left(\mathbb{I}^{\prime}{ }_{1}, \ldots, \mathbb{I}^{\prime}{ }_{n}\right)$ are entropy-based features, $h_{j}(\mathcal{T})$ is the value of pheromones, $\varepsilon_{j}$ shows the empirical value, $\dddot{w}$ is the cost of pheromones, $\mathcal{W}$ represents rational knowledge, and $\mathfrak{t}$ denotes the time limit. $h_{j}(T)$ and $\varepsilon_{j}$ are attached to the $\mathrm{j}^{\text {th }}$ feature. It is important to mention here that this will be considered an incomplete response if features are not studied up to this point.

\subsection{Dataset Balancing}

The MIT dataset contains a total of 888 images, out of which 600 are male images and 288 are female images. The number of male and female images is equal in MIT dataset; therefore, this dataset is an imbalanced dataset, leading to the following two research 
problems: (1) class imbalance problem, which results in poor performance, and (2) small sample space problem, which affects the training of model. Data balancing is selected to enhance the size of dataset and balancing of class-wise data. For this purpose, mirroring and horizontal flipping functions are applied. As a result, 264 male images and 576 female images are added to total 864 male and 864 female images. In this way, the size of MIT dataset is increased and, hence, class-wise data are also balanced.

\subsection{Classification}

After the selection of features, these are provided to the selected classifiers of SVM [64] and KNN [65] to perform classification. The SVM classifiers include linear variant [66] (LSVM), quadratic variant (QSVM) [67], fine Gaussian variant (FGSVM) [68], medium Gaussian variant (MGSVM), coarse Gaussian (CGSVM), and cubic variant (CSVM) [69]. The details of the kernels of these SVM classifiers can be found in [70-72]. The classifiers chosen from KNN include coarse variant (CRKNN), fine variant (FKNN), and cosine variant (COKNN) [73]. The details of these variants are available in [73-76]. The evaluation of classifiers is conducted on various performance evaluation metrics. Keeping in view the results obtained from experiments, it was observed that CSVM, QSVM, and FKNN classifiers produced better results. FKNN produced highest accuracy with MIT dataset, and CSVM was observed as best classifier in case of PKU-Reid dataset. The details of experiments performed and results produced are presented in the Results section.

\section{Results and Discussion}

This research was aimed to introduce a novel CNN network based on deep learning to classify pedestrian image datasets. A robust feature set was extracted with the proposed 4-BSMAB CNN-based network, and then various SVM and KNN classifiers were applied to obtain feature sets for evaluating the performance of system. The analysis and outcomes of proposed framework are presented in this section. In the first part, the details of experimental setup along with the datasets used and evaluation protocols applied are provided, and second part explains the experiments performed, which were carried out using a core i5 machine with Windows 10 platform, 8GB memory, and GPU (NVIDIA GTX 1070) with 8GB RAM (inbuilt). The MATLAB2020a tool was selected for programming purposes.

\subsection{Datasets}

Challenging datasets including viewpoint invariant pedestrian recognition (VIPeR) [77], pedestrian attribute (PETA) [78], cross-dataset [40], MIT [42], and Peking University reidentification (PKU-Reid) [79] were selected to test the proposed approach. Table 2 shows the details of these selected testing datasets. These datasets are also publicly available on the internet for experimental and research work. Different tasks related to pedestrian analysis such as person attribute analysis and PGC have been performed on these datasets. The challenges that exist in these datasets include inter- and intraclass variations (IICV), and environment recording settings (ERS). The IICV consist of speed and style of the movement of pedestrians, and ERS include pose variations, illumination changes, viewpoint changes, recording rates, camera settings, complex backgrounds, object deformation, shadow, and occlusion. Table 3 shows view-based information of testing datasets on which the evaluation of proposed model was carried out. 
Table 2. Statistics of testing datasets for PGC.

\begin{tabular}{ccccccc}
\hline $\begin{array}{c}\text { Sr. } \\
\text { No. }\end{array}$ & Datasets & Year & $\begin{array}{c}\text { \# Im- } \\
\text { ages/Videos }\end{array}$ & Views & Size & Applications \\
\hline 1 & VIPeR & 2008 & 1264 & $\begin{array}{c}\text { Side, Front, } \\
\text { Back }\end{array}$ & $128 \times 48$ & $\begin{array}{c}\text { Pedestrian } \\
\text { re-identification and } \\
\text { tracking across } \\
\text { multi-camera network }\end{array}$ \\
\hline 2 & MIT & 2014 & 888 & Front, Back & $128 \times 48$ & $\begin{array}{c}\text { Pedestrian attribute } \\
\text { analysis }\end{array}$ \\
\hline 3 & $\begin{array}{c}\text { PKU- } \\
\text { Reid }\end{array}$ & 2016 & 1824 & $\begin{array}{c}\text { Side, Front, } \\
\text { Back }\end{array}$ & $128 \times 48$ & $\begin{array}{c}\text { Pedestrian attribute } \\
\text { analysis and } \\
\text { re-identification }\end{array}$ \\
\hline
\end{tabular}

Table 3. View-based sample in MIT, VIPER, PKU-Reid, and PETA datasets used for testing of proposed model.

\begin{tabular}{ccccccc}
\hline \multirow{2}{*}{$\begin{array}{c}\text { Type of Views for } \\
\text { Testing }\end{array}$} & \multicolumn{2}{c}{ MIT } & \multicolumn{2}{c}{ VIPeR } & \multicolumn{2}{c}{ PKU-Reid } \\
\cline { 2 - 7 } & Male & Female & Male & Female & Male & Female \\
\hline Front & 305 & 113 & 339 & 251 & 420 & 264 \\
\hline Back & 296 & 174 & 198 & 133 & 140 & 88 \\
\hline Mixed & 864 & 864 & 721 & 543 & 1120 & 520 \\
\hline
\end{tabular}

In MIT dataset, 305 male images and 113 female images were selected for front viewbased evaluation, 296 male images and 174 female images were selected for backview-based evaluation, and 864 male and 864 female images were selected in case of mixed view-based evaluation. The VIPeR dataset contains 339 male images and 251 female images for front views, 198 male and 133 female images for back views, and 721 male images and 543 female images for mixed views. In PKU-Reid dataset, front views include 420 male and 264 female images, back views include 140 male and 88 female images, and mixed views include 1120 and 520 images of males and females, respectively. Figure 4 shows sample images of males as well as females taken from these datasets.

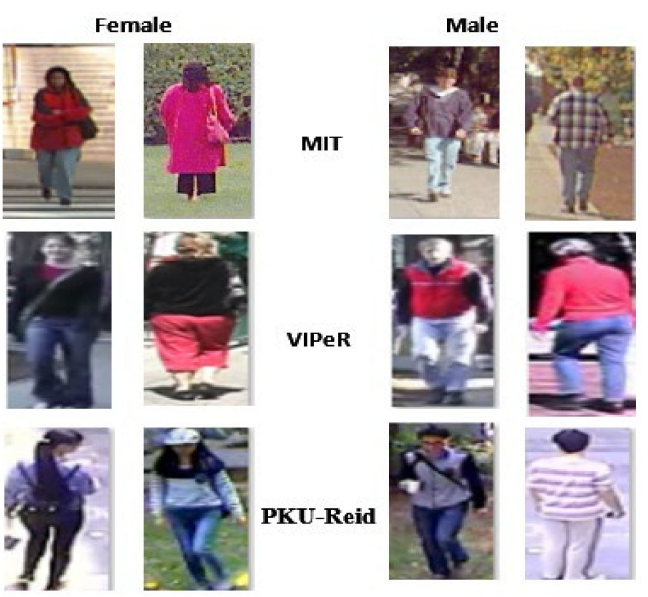

Figure 4. Some images of males and females from MIT, VIPeR, and PKU-Reid datasets.

\subsection{Performance Evaluation Protocols}

The evaluation of PGC problems directly relates to different accuracies and AUC. In this work, generally used performance evaluation metrics, i.e., accuracy (ACC), receiver operating characteristic (ROC) curve, F-measure (FM), G-measure (GM), area under the curve (AUC), true positive rate (TPR), and false positive rate (FPR), were selected for the measurement of performance of different PGC methods. Table 4 shows these metrics with 
their mathematical representation. Five-fold-type cross-validation was adopted for training and testing.

Table 4. Performance evaluation metrics.

\begin{tabular}{ccc}
\hline Sr. No. & Performance Measures & Mathematical Representation \\
\hline 1 & FPR & $\frac{F P}{T N+F P}$ \\
\hline 2 & Sensitivity (SE), TPR, Recall & $\frac{T P}{T P+F N}$ \\
\hline 3 & Specificity (SP), TNR & $\frac{T N}{T N+F P}$ \\
\hline 4 & Precision (PR) & $\frac{T P}{T P+F P}$ \\
\hline 5 & Accuracy (ACC) & $\frac{T P+T N}{T P+T N+F P+F N}$ \\
\hline 6 & AUC & $\int_{\infty}^{-\infty} T P R(t) F P R^{\prime}(t) d t$ \\
\hline 7 & F-Measure (FM) & $2=\frac{\text { Precision X Recall }}{\text { Precision X Recall }}$ \\
\hline 8 & G-Measure (GM) & $\sqrt{T P R X \text { TNR }}$ \\
\hline
\end{tabular}

\subsection{Performance Evaluation of Proposed Framework}

Experiments were performed with the proposed framework on MIT, VIPeR, and PKU-Reid testing datasets to achieve best results. For this purpose, various experiments were carried out with several variations of optimized feature subsets. A major analysis of these experiments is presented in this section. Table 5 shows the summary of experiments performed and accuracies produced by them with five feature subsets on front views, back views, and mixed views of selected datasets.

Table 5. Optimized feature subsets with dimensions and best accuracy on MIT, VIPeR, and PKU-Reid datasets.

\begin{tabular}{|c|c|c|c|c|c|c|c|c|c|c|}
\hline \multirow{2}{*}{$\begin{array}{l}\text { Optimized } \\
\text { Feature } \\
\text { Subset } \\
\text { No. }\end{array}$} & \multirow{2}{*}{$\begin{array}{l}\text { No. of } \\
\text { Features }\end{array}$} & \multicolumn{3}{|c|}{$\begin{array}{c}\text { Best ACC (\%) Achieved on MIT } \\
\text { Dataset }\end{array}$} & \multicolumn{3}{|c|}{$\begin{array}{c}\text { Best ACC (\%) Achieved on } \\
\text { VIPeR Dataset }\end{array}$} & \multicolumn{3}{|c|}{$\begin{array}{l}\text { Best ACC (\%) Achieved on } \\
\text { PKU-Reid Dataset }\end{array}$} \\
\hline & & $\begin{array}{l}\text { Front } \\
\text { Views }\end{array}$ & $\begin{array}{c}\text { Back } \\
\text { Views }\end{array}$ & $\begin{array}{l}\text { Mixed } \\
\text { Views }\end{array}$ & $\begin{array}{l}\text { Front } \\
\text { Views }\end{array}$ & $\begin{array}{c}\text { Back } \\
\text { Views }\end{array}$ & $\begin{array}{l}\text { Mixed } \\
\text { Views }\end{array}$ & $\begin{array}{l}\text { Front } \\
\text { Views }\end{array}$ & $\begin{array}{c}\text { Back } \\
\text { Views }\end{array}$ & $\begin{array}{l}\text { Mixed } \\
\text { Views }\end{array}$ \\
\hline 1 & 100 & 74.9 & 72.8 & 81.3 & 65.9 & 70.0 & 64.1 & 79.1 & 86.8 & 81.8 \\
\hline 2 & 250 & 74.6 & 73.4 & 84.6 & 67.0 & 66.5 & 68.4 & 85.7 & 88.6 & 88.0 \\
\hline 3 & 500 & 74.7 & 73.0 & 84.7 & 65.1 & 69.5 & 68.3 & 83.8 & 89.9 & 89.8 \\
\hline 4 & 750 & 74.9 & 73.8 & 85.1 & 69.3 & 72.5 & 69.5 & 84.2 & 90.4 & 91.2 \\
\hline 5 & 1000 & 74.9 & 73.8 & 85.4 & 72.9 & 70.7 & 70.3 & 85.5 & 93.0 & 91.2 \\
\hline
\end{tabular}

The fitness value graphs obtained by ACS on mixed views of MIT dataset are depicted in Figure 5. The fitness was maintained after 49 th iteration, with a value of 0.29 , by using 1000-feature subset.

\subsubsection{Performance Evaluation of MIT Dataset}

In this section, the results generated by experiments performed using front views, back views, and mixed view images of MIT testing dataset are mentioned. Five-fold-type cross-validation was utilized on all feature matrices of MIT dataset obtained from frontal views, back views, and mixed views of MIT dataset and provided to classifiers that are variants of KNN and SVM for automatic labeling. The details of the evaluation of proposed model with five different feature subsets on MIT testing dataset are presented below.

Evaluation of frontal views of MIT dataset: The best result achieved in terms of accuracy was $74.9 \%$ by LSVM with 1000-feature subset, CSVM with 750-feature subset, and QSVM with 1000-feature subset, while the second best result attained in terms of accuracy was $74.7 \%$ by LSVM with 500 -feature subset, as shown in Table 6 . The training 
time and prediction speed of proposed model on front views of MIT dataset are presented in Figure 6.

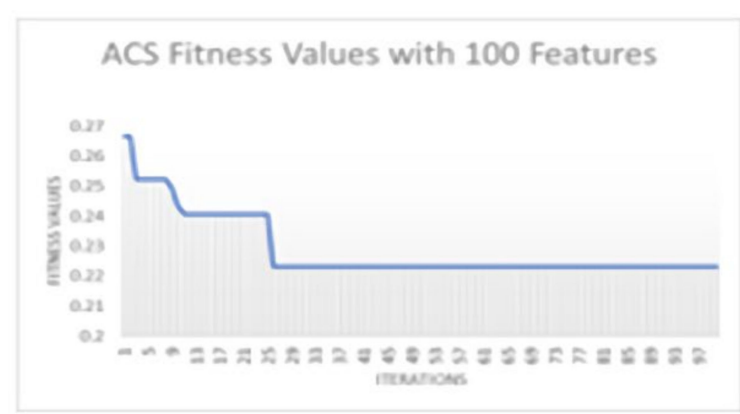

(a)

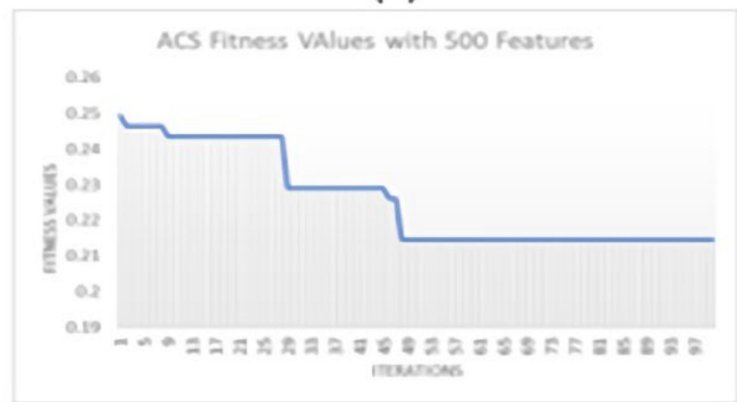

(c)

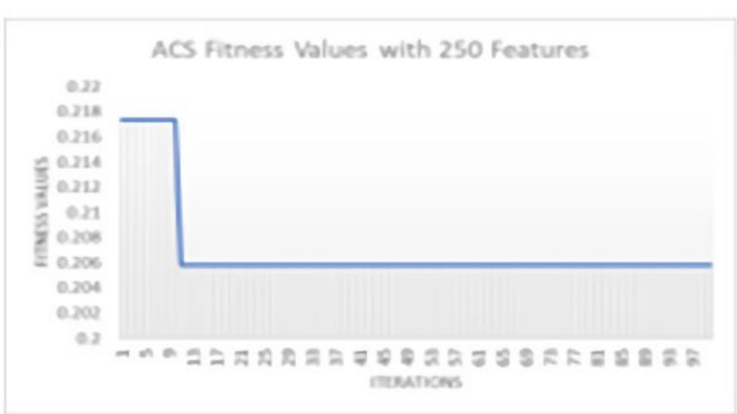

(b)

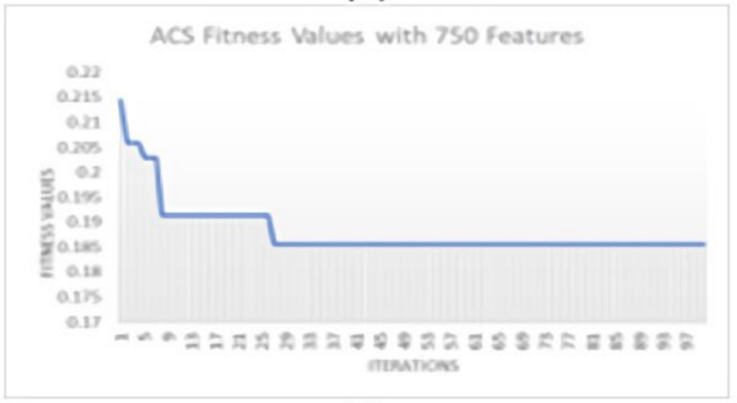

(d)

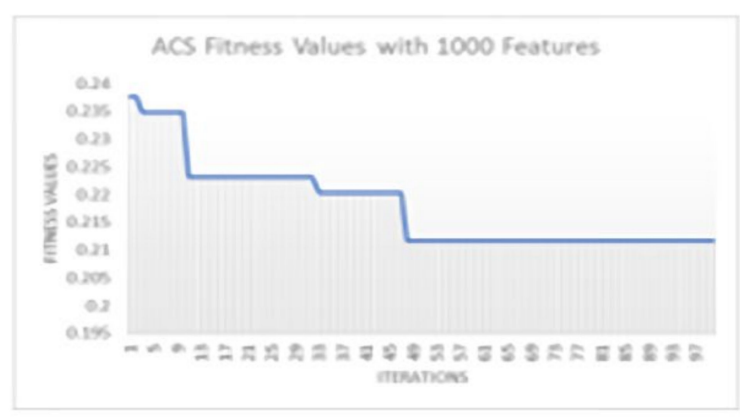

(e)

Figure 5. Fitness values of ACS-based optimization with (a) 100-feature, (b) 250-feature, (c) 500-feature, (d) 750-feature, and (e) 1000-feature subsets.

Table 6. Front view-based experimental results on MIT dataset.

\begin{tabular}{|c|c|c|c|c|c|c|c|c|c|c|c|c|}
\hline \multirow{2}{*}{$\begin{array}{l}\text { Classification } \\
\text { Methods }\end{array}$} & \multicolumn{5}{|c|}{ Optimized Feature Subsets } & \multicolumn{7}{|c|}{ Evaluation Metrics } \\
\hline & 100 & 250 & 500 & 750 & 1000 & $\mathrm{ACC}$ & AUC & SE & SP & PR & FM & GM \\
\hline LSVM & & & & & $\checkmark$ & 74.9 & 0.70 & 99.3 & 08.9 & 74.6 & 85.2 & 29.7 \\
\hline CSVM & & & & $\checkmark$ & & 74.9 & 0.71 & 91.8 & 29.2 & 77.8 & 84.2 & 51.8 \\
\hline MGSVM & & $\checkmark$ & & & & 74.0 & 0.70 & 100.0 & 03.5 & 74.7 & 84.8 & 18.8 \\
\hline QSVM & $\checkmark$ & & & & & 74.9 & 0.71 & 92.5 & 27.4 & 77.5 & 84.3 & 50.4 \\
\hline QSVM & & & & & $\checkmark$ & 74.9 & 0.71 & 92.5 & 27.4 & 77.5 & 84.3 & 50.4 \\
\hline FGSVM & & & & & $\checkmark$ & 73.7 & 0.52 & 100.0 & 02.7 & 73.5 & 84.7 & 16.3 \\
\hline CGSVM & & & & & $\checkmark$ & 73.2 & 0.69 & 100.0 & 00.9 & 73.1 & 84.5 & 09.4 \\
\hline FKNN & & & & $\checkmark$ & & 68.9 & 0.54 & 82.3 & 32.7 & 76.8 & 79.4 & 52.0 \\
\hline COKNN & & & $\checkmark$ & & & 73.2 & 0.59 & 96.1 & 11.5 & 73.6 & 84.0 & 33.2 \\
\hline COKNN & & & & & $\checkmark$ & 73.2 & 0.59 & 95.7 & 12.4 & 74.7 & 84.0 & 34.4 \\
\hline CRKNN & & & & & $\checkmark$ & 73.0 & 0.65 & 100.0 & 00.0 & 73.0 & 84.4 & 00.0 \\
\hline
\end{tabular}




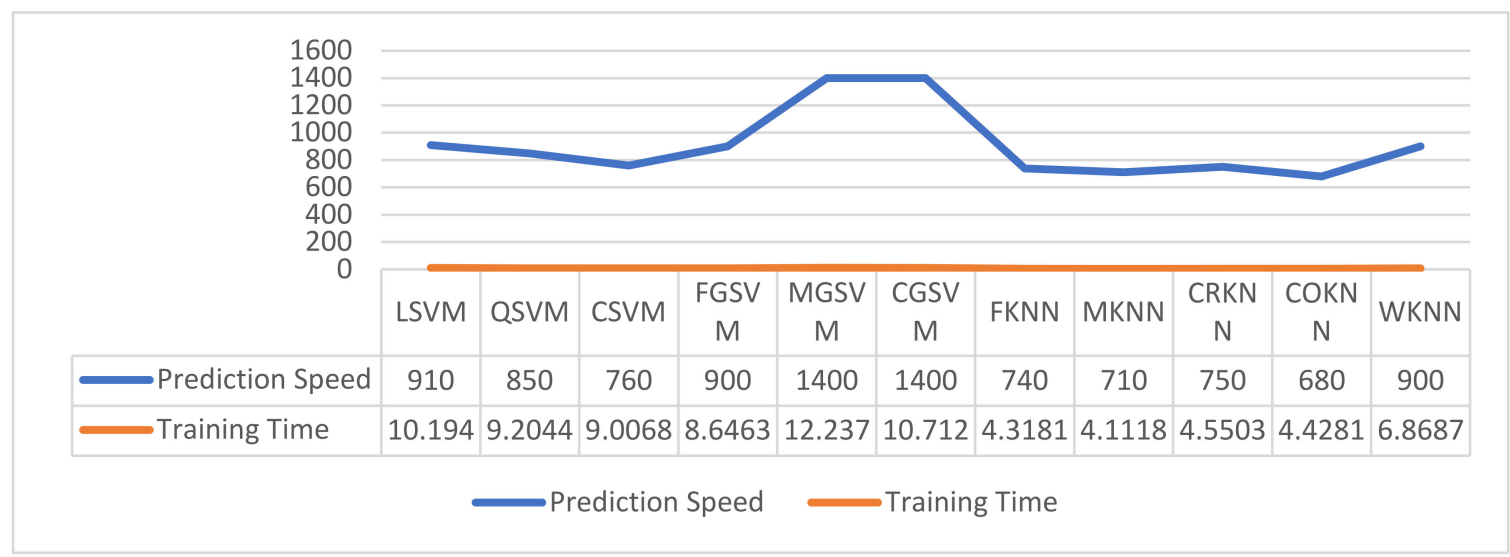

Figure 6. Proposed model training time (s) and prediction speed (obs/s) on front views of MIT dataset utilizing 1000feature subset.

Evaluation of back views of MIT dataset: The best accuracy achieved on MIT dataset was $73.8 \%$ by CSVM classifier with 1000-feature subset, and QSVM with 750-feature subset, while the second best result obtained in terms of accuracy was $73.4 \%$ by QSVM with 250-feature subset, as shown in Table 7 . The training time and prediction speed of proposed model on back views of MIT dataset are presented in Figure 7.

Table 7. Back view-based experimental results on MIT dataset.

\begin{tabular}{|c|c|c|c|c|c|c|c|c|c|c|c|c|}
\hline \multirow{2}{*}{$\begin{array}{l}\text { Classification } \\
\text { Methods }\end{array}$} & \multicolumn{5}{|c|}{ Optimized Feature Subsets } & \multicolumn{7}{|c|}{ Evaluation Metrics } \\
\hline & 100 & 250 & 500 & 750 & 1000 & $\mathrm{ACC}$ & AUC & SE & SP & PR & FM & GM \\
\hline LSVM & & & $\checkmark$ & & & 73.0 & 0.77 & 91.2 & 42.0 & 72.8 & 81.0 & 61.9 \\
\hline CSVM & & & & & $\checkmark$ & 73.8 & 0.79 & 85.1 & 54.6 & 76.1 & 80.4 & 68.2 \\
\hline MGSVM & & $\checkmark$ & & & & 70.7 & 0.78 & 94.3 & 305 & 69.8 & 80.2 & 536 \\
\hline QSVM & & & & $\checkmark$ & & 73.8 & 0.79 & 83.8 & 56.9 & 76.8 & 80.1 & 69.0 \\
\hline FGSVM & & & $\checkmark$ & & & 63.0 & 0.53 & 100.0 & 00.0 & 63.0 & 77.3 & 00.0 \\
\hline CGSVM & & & & & $\checkmark$ & 65.8 & 0.77 & 99.7 & 08.1 & 64.8 & 78.6 & 28.3 \\
\hline FKNN & & & & $\checkmark$ & & 65.1 & 0.58 & 74.7 & 48.9 & 71.3 & 73.0 & 60.4 \\
\hline COKNN & & $\checkmark$ & & & & 68.3 & 0.66 & 84.5 & 40.8 & 70.8 & 77.0 & 58.7 \\
\hline CRKNN & & & & $\checkmark$ & & 63.4 & 0.70 & 100.0 & 01.2 & 63.3 & 77.5 & 10.7 \\
\hline
\end{tabular}

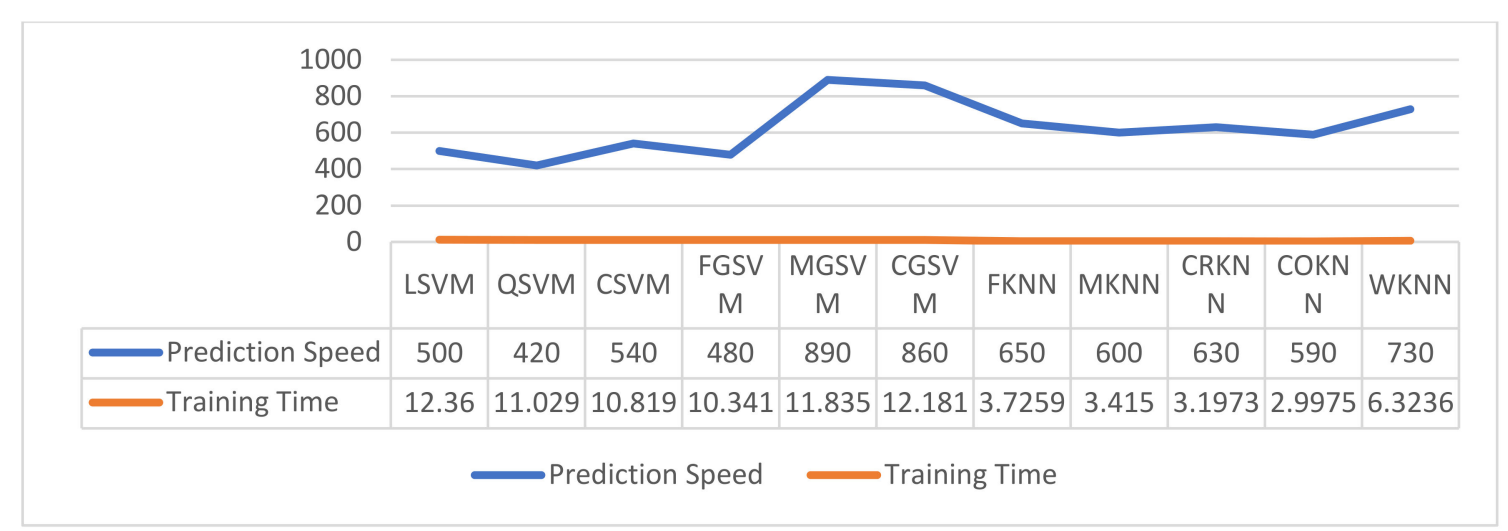

Figure 7. Proposed model training time (s) and prediction speed (obs/s) on back views of MIT dataset utilizing 1000feature subset. 
Evaluation of mixed views of MIT dataset: The best result achieved in terms of accuracy was $85.3 \%$ by FKNN with 1000 -feature subset, while the second best result attained in terms of accuracy was $85.1 \%$ by FKNN with 750 -feature subset, as shown in Table 8 . The training time and prediction speed of proposed model on mixed views of MIT dataset are presented in Figure 8.

Table 8. Mixed view-based experimental results on MIT dataset.

\begin{tabular}{|c|c|c|c|c|c|c|c|c|c|c|c|c|}
\hline \multirow{2}{*}{$\begin{array}{l}\text { Classification } \\
\text { Methods }\end{array}$} & \multicolumn{5}{|c|}{ Optimized Feature Subsets } & \multicolumn{7}{|c|}{ Evaluation Metrics } \\
\hline & 100 & 250 & 500 & 750 & 1000 & Acc & Auc & Se & $\mathrm{Sp}$ & PR & FM & GM \\
\hline LSVM & & & & $\checkmark$ & & 76.9 & 0.84 & 77.0 & 76.8 & 77.0 & 76.9 & 76.9 \\
\hline CSVM & & & & $\checkmark$ & & 83.9 & 0.92 & 82.6 & 85.1 & 84.7 & 83.7 & 83.9 \\
\hline MGSVM & & & & $\checkmark$ & & 81.9 & 0.89 & 88.5 & 75.1 & 78.1 & 83.0 & 81.6 \\
\hline QSVM & & & & $\checkmark$ & & 81.7 & 0.89 & 81.3 & 82.1 & 81.9 & 81.6 & 81.7 \\
\hline FGSVM & $\checkmark$ & & & & & 67.3 & 0.66 & 95.1 & 39.4 & 61.1 & 74.4 & 61.2 \\
\hline CGSVM & & & & $\checkmark$ & & 75.5 & 0.80 & 76.7 & 74.2 & 74.8 & 75.8 & 75.5 \\
\hline FKNN & & & & & $\checkmark$ & 85.4 & 0.89 & 79.9 & 90.7 & 89.6 & 84.5 & 85.1 \\
\hline COKNN & & & & $\checkmark$ & & 73.7 & 0.81 & 77.3 & 70.0 & 72.1 & 74.6 & 73.6 \\
\hline CRKNN & & & $\checkmark$ & & & 67.8 & 0.74 & 75.9 & 59.6 & 65.3 & 70.2 & 67.3 \\
\hline
\end{tabular}

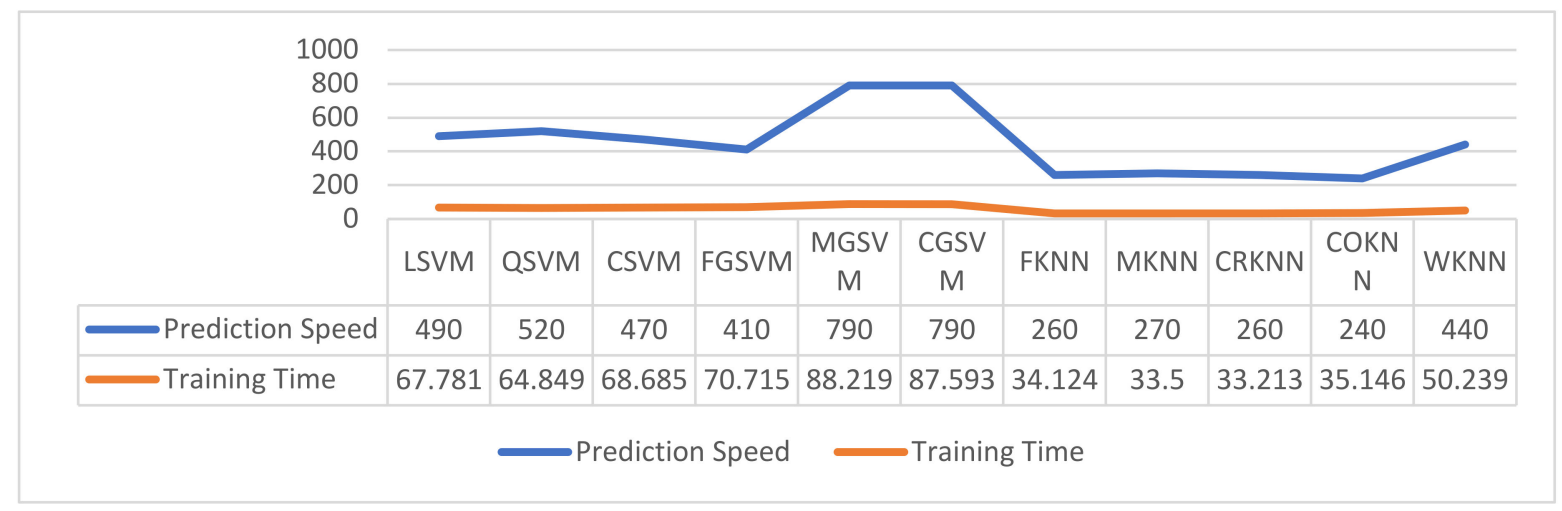

Figure 8. Proposed model training time (s) and prediction speed (obs/s) on mixed views of MIT dataset utilizing 1000feature subset.

The best ROC outcomes on MIT dataset are presented in Figure 9.

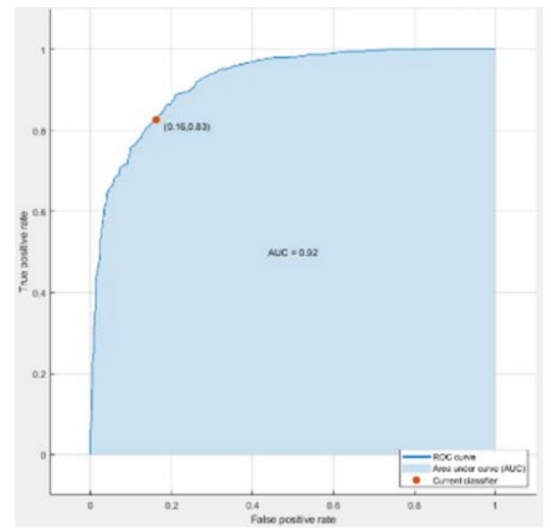

(a)

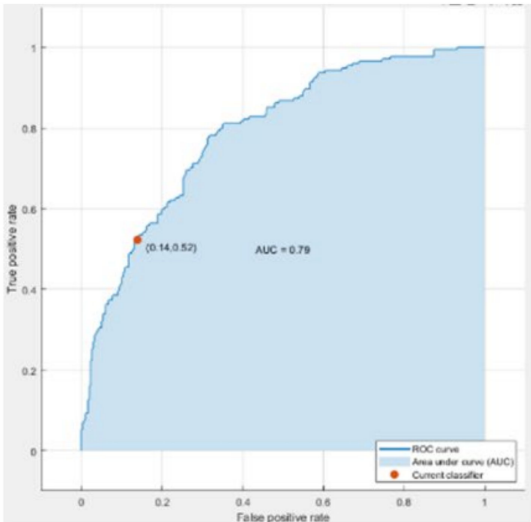

(b)

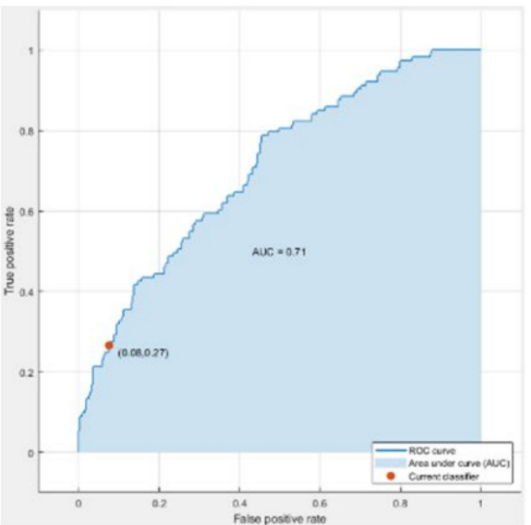

(c)

Figure 9. ROC curves and AUC values showing best outcomes of (a) mixed views with CSVM, (b) back views with QSVM, and (c) front views with QSVM of MIT dataset utilizing 1000-feature subset. 


\subsubsection{Performance Evaluation of VIPeR Dataset}

In this section, the results generated by experiments performed using front views, back views, and mixed view images of VIPeR testing dataset are mentioned. Five-fold-type cross-validation was utilized on all feature matrices of MIT dataset obtained from frontal views, back views, and mixed views of VIPeR dataset and provided to classifiers that are variants of KNN and SVM for automatic labeling. The details of the evaluation of proposed model with five different feature subsets on VIPeR testing dataset are presented below.

Evaluation of frontal views of VIPeR dataset: The best result achieved in terms of accuracy was $72.9 \%$ by QSVM with 1000 -feature subset, while the second best result obtained in terms of accuracy was $70.7 \%$ by CSVM with 1000 -feature subset, as shown in Table 9. The training time and prediction speed of proposed model on front views of VIPeR dataset are presented in Figure 10.

Table 9. Front view-based experimental results on VIPeR dataset.

\begin{tabular}{|c|c|c|c|c|c|c|c|c|c|c|c|c|}
\hline \multirow{2}{*}{$\begin{array}{l}\text { Classification } \\
\text { Methods }\end{array}$} & \multicolumn{5}{|c|}{ Optimized Feature Subsets } & \multicolumn{7}{|c|}{ Evaluation Metrics } \\
\hline & 100 & 250 & 500 & 750 & 1000 & ACC & AUC & SE & SP & PR & FM & GM \\
\hline LSVM & & & & $\checkmark$ & & 69.3 & 0.76 & 82.3 & 51.8 & 69.8 & 75.5 & 65.3 \\
\hline CSVM & & & & & $\checkmark$ & 70.7 & 0.75 & 78.8 & 59.8 & 72.6 & 75.5 & 68.6 \\
\hline MGSVM & & $\checkmark$ & & & & 67.0 & 0.76 & 81.7 & 47.0 & 67.6 & 74.0 & 62.0 \\
\hline QSVM & & & & & $\checkmark$ & 72.9 & 0.76 & 78.8 & 64.9 & 75.2 & 77.0 & 71.5 \\
\hline FGSVM & & & $\checkmark$ & & & 57.5 & 0.57 & 100.0 & 00.0 & 57.5 & 73.0 & 00.0 \\
\hline CGSVM & & & & & $\checkmark$ & 66.8 & 0.74 & 91.5 & 33.5 & 65.0 & 76.0 & 55.3 \\
\hline FKNN & & & & $\checkmark$ & & 60.3 & 0.61 & 69.6 & 47.8 & 64.3 & 66.9 & 57.7 \\
\hline COKNN & & & & & $\checkmark$ & 64.8 & 0.68 & 80.2 & 43.8 & 65.9 & 72.3 & 59.3 \\
\hline CRKNN & & & & & $\checkmark$ & 66.8 & 0.69 & 91.5 & 33.5 & 65.0 & 76.0 & 553 \\
\hline
\end{tabular}

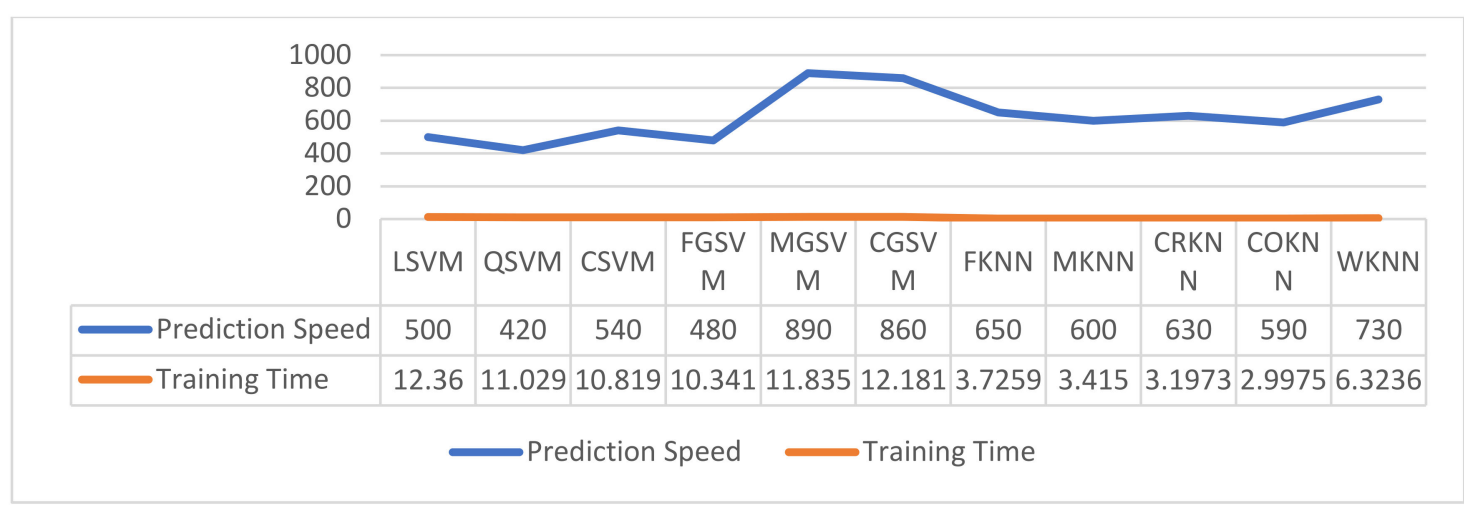

Figure 10. Proposed model training time (s) and prediction speed (obs/s) on front views of VIPeR dataset utilizing 1000-feature subset.

Evaluation of back views of VIPeR dataset: The best result achieved in terms of accuracy was $72.5 \%$ by QSVM with 750-feature subset, while the second best result attained in terms of accuracy was $70.7 \%$ by LSVM with 1000 -feature subset, as shown in Table 10. The training time and prediction speed of proposed model on back views of VIPeR dataset are presented in Figure 11. 
Table 10. Back view-based experimental results on VIPeR dataset.

\begin{tabular}{|c|c|c|c|c|c|c|c|c|c|c|c|c|}
\hline \multirow{2}{*}{$\begin{array}{l}\text { Classification } \\
\text { Methods }\end{array}$} & \multicolumn{5}{|c|}{ Optimized Feature Subsets } & \multicolumn{7}{|c|}{ Evaluation Metrics } \\
\hline & 100 & 250 & 500 & 750 & 1000 & ACC & AUC & SE & SP & PR & FM & GM \\
\hline LSVM & & & & & $\checkmark$ & 70.7 & 0.76 & 86.9 & 46.6 & 70.8 & 78.0 & 63.7 \\
\hline CSVM & $\checkmark$ & & & & & 70.0 & 0.78 & 80.3 & 54.1 & 72.3 & 76.1 & 65.9 \\
\hline MGSVM & $\checkmark$ & & & & & 68.9 & 0.76 & 92.9 & 33.1 & 67.4 & 78.1 & 55.5 \\
\hline QSVM & & & & $\checkmark$ & & 72.5 & 0.78 & 82.8 & 57.1 & 74.2 & 78.3 & 68.8 \\
\hline FGSVM & & & $\checkmark$ & & & 59.8 & 0.51 & 100.0 & 00.0 & 59.8 & 74.9 & 00.0 \\
\hline CGSVM & & & & & $\checkmark$ & 61.9 & 0.75 & 99.5 & 60.2 & 61.2 & 75.8 & 24.5 \\
\hline FKNN & & & $\checkmark$ & & & 58.3 & 0.75 & 68.2 & 43.6 & 64.3 & 66.2 & 54.5 \\
\hline FKNN & & & & & $\checkmark$ & 58.3 & 0.75 & 64.7 & 48.9 & 65.3 & 65.0 & 56.2 \\
\hline COKNN & & & & & $\checkmark$ & 65.0 & 0.65 & 79.3 & 43.6 & 67.7 & 73.0 & 58.8 \\
\hline CRKNN & & & & & $\checkmark$ & 61.3 & 0.71 & 99.5 & 04.5 & 60.8 & 75.5 & 21.2 \\
\hline
\end{tabular}

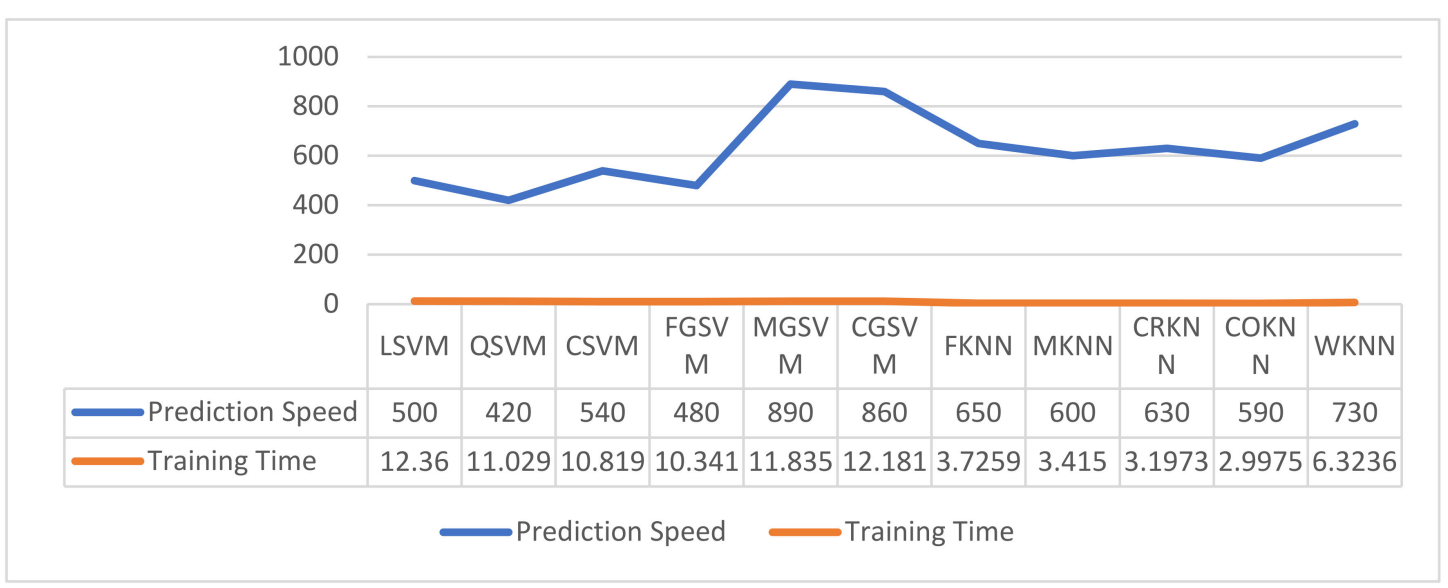

Figure 11. Proposed model training time (s) and prediction speed (obs/s) on back views of VIPeR dataset utilizing 1000-feature subset.

Evaluation of mixed views of VIPeR dataset: The best accuracy achieved was $70.3 \%$ by CSVM with 1000-feature subset, while the second best result obtained in terms of accuracy was $69.5 \%$ by LSVM with 750 -feature subset, as shown in Table 11 . The training time and prediction speed of proposed model on mixed views of VIPeR dataset are presented in Figure 12. The best ROC outcomes on VIPeR dataset are presented in Figure 13.

Table 11. Mixed view-based experimental results on VIPeR dataset.

\begin{tabular}{|c|c|c|c|c|c|c|c|c|c|c|c|c|}
\hline \multirow{2}{*}{$\begin{array}{l}\text { Classification } \\
\text { Methods }\end{array}$} & \multicolumn{5}{|c|}{ Optimized Feature Subsets } & \multicolumn{7}{|c|}{ Evaluation Metrics } \\
\hline & 100 & 250 & 500 & 750 & 1000 & $\mathrm{ACC}$ & AUC & SE & SP & PR & FM & GM \\
\hline LSVM & & & & $\checkmark$ & & 69.5 & 0.74 & 80.2 & 55.4 & 70.5 & 75.0 & 66.7 \\
\hline CSVM & & & & & $\checkmark$ & 70.3 & 0.74 & 78.0 & 60.2 & 72.2 & 75.0 & 68.5 \\
\hline MGSVM & & & $\checkmark$ & & & 68.3 & 0.75 & 84.5 & 46.8 & 67.8 & 75.2 & 62.9 \\
\hline QSVM & & & & & $\checkmark$ & 69.1 & 0.74 & 78.0 & 57.3 & 70.8 & 74.2 & 66.9 \\
\hline FGSVM & & & $\checkmark$ & & & 57.0 & 0.53 & 1.00 & 0.00 & 57.4 & 72.7 & 0.00 \\
\hline CGSVM & & & & $\checkmark$ & & 68.4 & 0.73 & 88.0 & 42.5 & 67.0 & 76.1 & 61.2 \\
\hline CGSVM & & & & & $\checkmark$ & 68.4 & 0.73 & 83.0 & 49.2 & 68.4 & 75.0 & 63.9 \\
\hline FKNN & $\checkmark$ & & & & & 58.4 & 0.56 & 66.9 & 47.2 & 62.7 & 64.7 & 56.1 \\
\hline FKNN & & $\checkmark$ & & & & 58.4 & 0.56 & 66.9 & 47.2 & 62.7 & 64.7 & 56.0 \\
\hline COKNN & & & & $\checkmark$ & & 63.2 & 0.66 & 72.9 & 50.5 & 66.1 & 69.3 & 60.7 \\
\hline CRKNN & & & $\checkmark$ & & & 60.4 & 0.69 & 98.5 & 09.8 & 59.2 & 74.0 & 31.0 \\
\hline
\end{tabular}




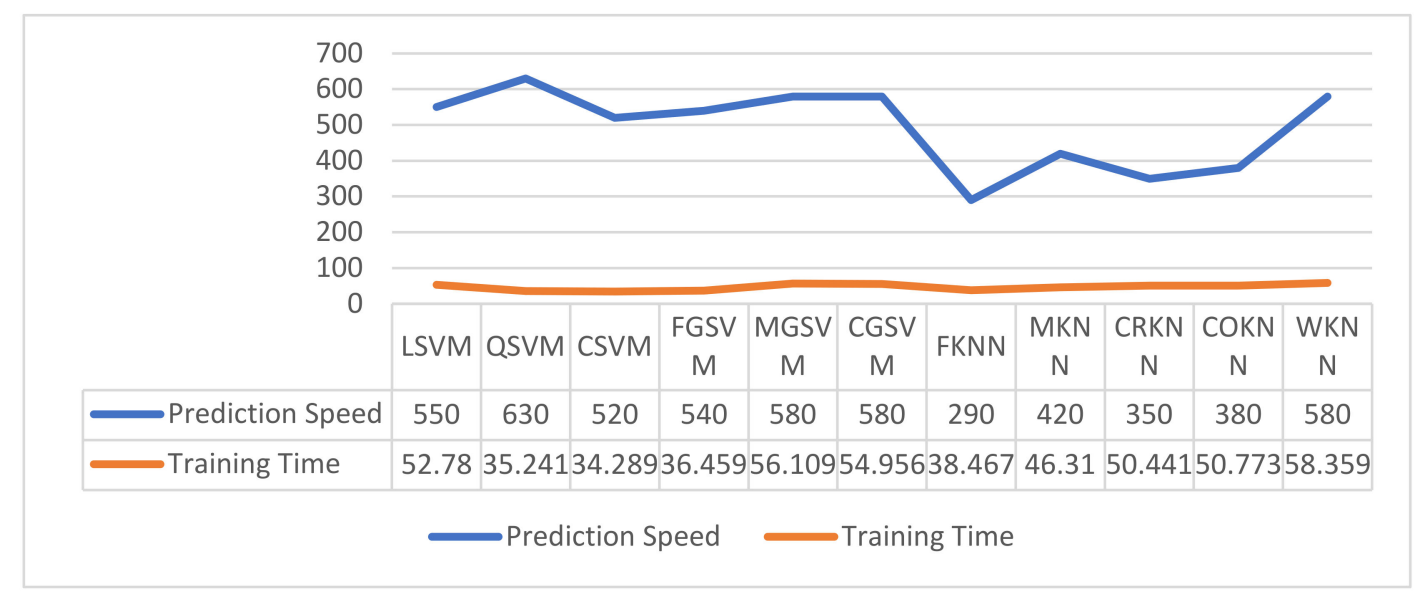

Figure 12. Proposed model training time (s) and prediction speed (obs/s) on mixed views of VIPeR dataset utilizing 1000-feature subset.

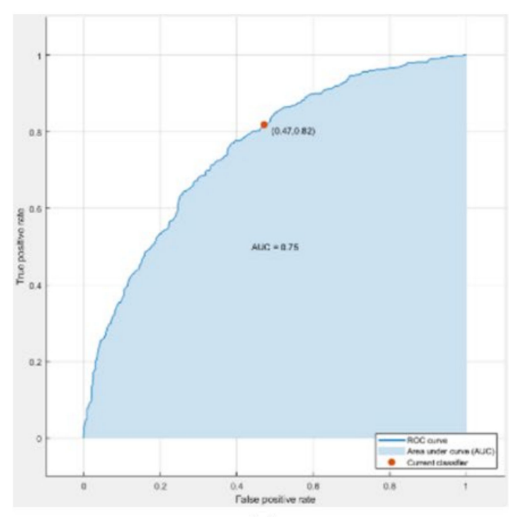

(a)

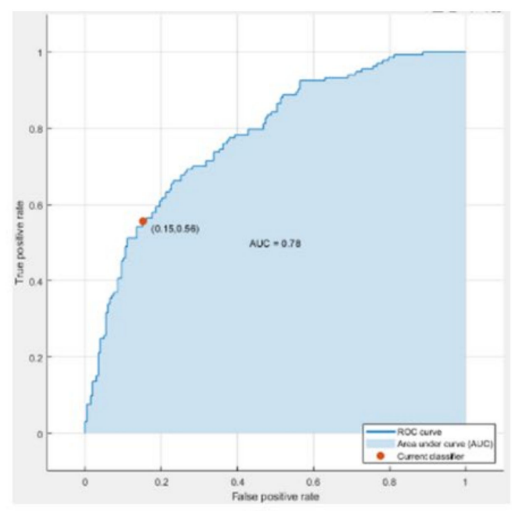

(b)

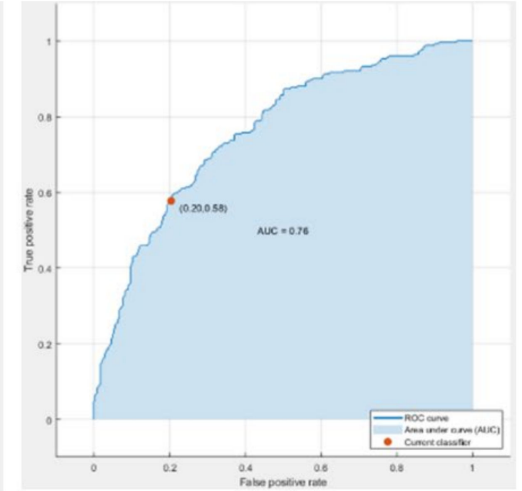

(c)

Figure 13. ROC curves and AUC values showing best outcomes of (a) mixed views with MGVM, (b) back views with CSVM, and (c) front views with QSVM of IPeR dataset utilizing 1000-feature subset.

\subsubsection{Performance Evaluation of PKU-Reid Dataset}

This section describes the results generated by experiments performed using front views, back views, and mixed view images of PKU-Reid testing dataset. Five-fold-type cross-validation was utilized on all feature matrices of MIT dataset obtained from frontal views, back views, and mixed views of PKU-Reid dataset and provided to classifiers that are variants of KNN and SVM for automatic labeling. The details of the evaluation of proposed model with five different feature subsets on PKU-Reid testing dataset are presented below.

Evaluation of frontal views of PKU-Reid dataset: The best accuracy achieved was $85.7 \%$ by CSVM with 250-featuresubset, while the second best accuracy attained was $85.5 \%$ by CSVM with 1000-feature subset, as shown in Table 12. The training time and prediction speed of proposed model on front views of PKU-Reid dataset are presented in Figure 14. 
Table 12. Front view-based experimental results on PKU-Reid dataset.

\begin{tabular}{|c|c|c|c|c|c|c|c|c|c|c|c|c|}
\hline \multirow{2}{*}{$\begin{array}{l}\text { Classification } \\
\text { Methods }\end{array}$} & \multicolumn{5}{|c|}{ Optimized Feature Subsets } & \multicolumn{7}{|c|}{ Evaluation Metrics } \\
\hline & 100 & 250 & 500 & 750 & 1000 & $\mathrm{ACC}$ & AUC & SE & SP & PR & FM & GM \\
\hline LSVM & & & & & $\checkmark$ & 82.9 & 0.91 & 91.2 & 69.7 & 82.7 & 86.8 & 79.7 \\
\hline CSVM & & $\checkmark$ & & & & 85.7 & 0.93 & 91.2 & 76.9 & 86.3 & 88.7 & 83.7 \\
\hline MGSVM & & $\checkmark$ & & & & 82.2 & 0.92 & 92.9 & 65.2 & 80.9 & 86.5 & 77.8 \\
\hline QSVM & & & & $\checkmark$ & & 84.2 & 0.92 & 89.5 & 75.8 & 85.5 & 87.4 & 82.4 \\
\hline FGSVM & & & $\checkmark$ & & & 61.4 & 0.65 & 100.0 & 00.0 & 61.4 & 76.1 & 00.0 \\
\hline CGSVM & & & & & $\checkmark$ & 82.3 & 0.89 & 94.8 & 62.5 & 80.1 & 86.8 & 77.0 \\
\hline FKNN & & & & & $\checkmark$ & 74.3 & 0.71 & 85.2 & 56.8 & 75.9 & 80.3 & 69.6 \\
\hline COKNN & & & & $\checkmark$ & & 78.1 & 0.84 & 89.3 & 60.2 & 78.1 & 83.3 & 73.3 \\
\hline CRKNN & $\checkmark$ & & & & & 66.1 & 0.84 & 99.5 & 12.9 & 64.5 & 78.3 & 35.8 \\
\hline CRKNN & & & & $\checkmark$ & & 66.1 & 0.84 & 98.8 & 14.0 & 64.6 & 78.2 & 37.2 \\
\hline
\end{tabular}

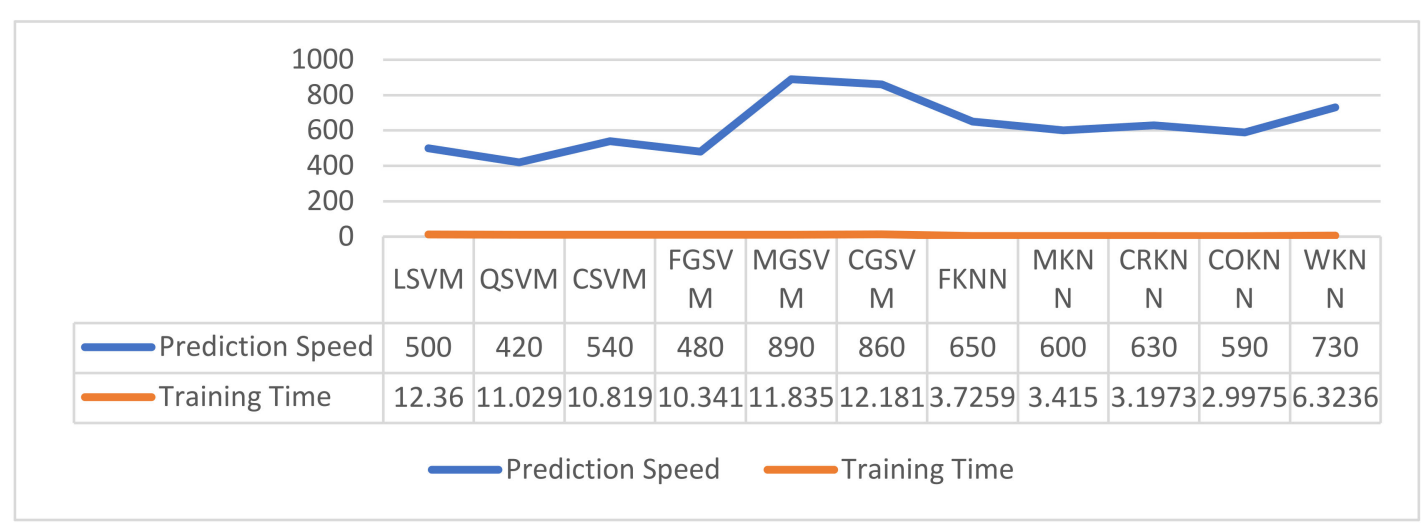

Figure 14. Proposed model training time (s) and prediction speed (obs/s) on front views of PKU-Reid dataset utilizing 1000-feature subset.

Evaluation of back views of PKU-Reid dataset: The best accuracy achieved was 93.0\% by CSVM with 1000-feature subset, while the second best result obtained in terms of accuracy was $92.5 \%$ by QSVM with 1000 -feature subset, as shown in Table 13 . The training time and prediction speed of proposed model on back views of PKU-Reid dataset are presented in Figure 15.

Table 13. Back view-based experimental results on PKU-Reid dataset.

\begin{tabular}{|c|c|c|c|c|c|c|c|c|c|c|c|c|}
\hline \multirow{2}{*}{$\begin{array}{l}\text { Classification } \\
\text { Methods }\end{array}$} & \multicolumn{5}{|c|}{ Optimized Feature Subsets } & \multicolumn{7}{|c|}{ Evaluation Metrics } \\
\hline & 100 & 250 & 500 & 750 & 1000 & ACC & AUC & SE & SP & PR & FM & GM \\
\hline LSVM & & & & $\checkmark$ & & 90.4 & 0.96 & 97.1 & 79.6 & 88.3 & 92.5 & 87.9 \\
\hline CSVM & & & & & $\checkmark$ & 93.0 & 0.96 & 97.9 & 85.2 & 91.3 & 94.5 & 91.3 \\
\hline MGSVM & $\checkmark$ & & & & & 86.0 & 0.95 & 96.4 & 69.3 & 83.3 & 89.4 & 81.8 \\
\hline QSVM & & & & & $\checkmark$ & 92.5 & 0.96 & 97.1 & 85.2 & 91.3 & 94.1 & 91.0 \\
\hline FGSVM & & & $\checkmark$ & & & 61.4 & 0.59 & 100.0 & 00.0 & 61.4 & 76.1 & 00.0 \\
\hline CGSVM & & & & & $\checkmark$ & 87.7 & 0.94 & 99.3 & 69.3 & 83.7 & 90.9 & 83.0 \\
\hline FKNN & & & & $\checkmark$ & & 75.4 & 0.72 & 90.7 & 51.1 & 74.7 & 82.0 & 68.1 \\
\hline COKNN & & & & & $\checkmark$ & 80.7 & 0.89 & 92.1 & 62.5 & 79.6 & 85.4 & 75.9 \\
\hline CRKNN & & & $\checkmark$ & & & 61.4 & 0.85 & 100.0 & 00.0 & 61.4 & 76.1 & 00.0 \\
\hline
\end{tabular}




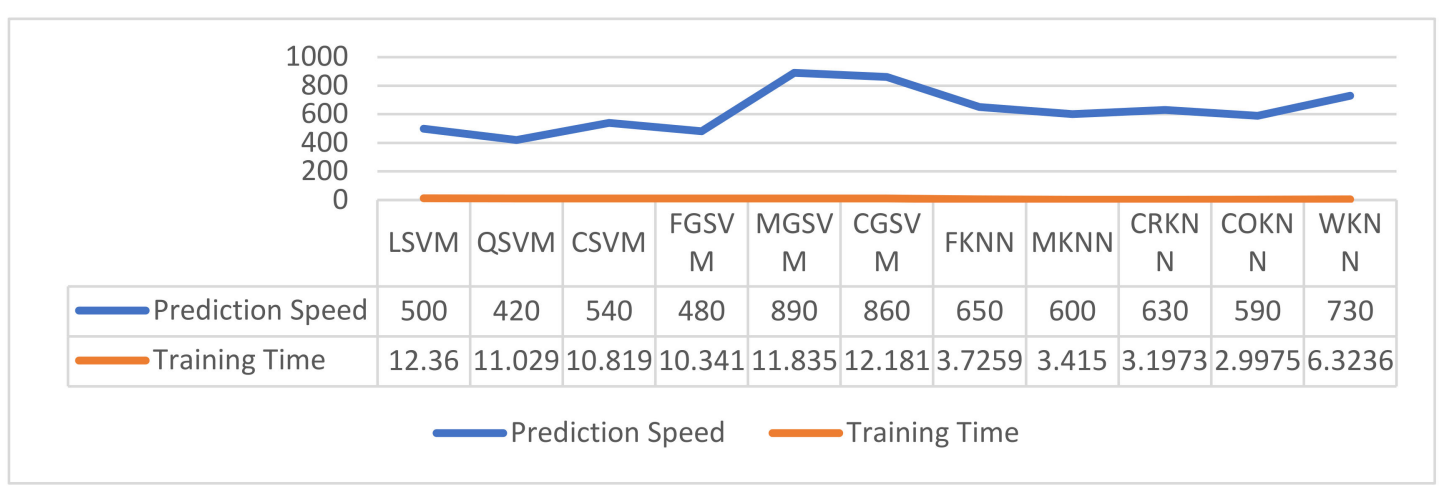

Figure 15. Proposed model training time (s) and prediction speed (obs/s) on back views of PKU-Reid dataset utilizing 1000-feature subset.

Evaluation of mixed views of PKU-Reid dataset: The best accuracy achieved was 91.2\% by CSVM with 750- and 1000-feature subsets, while the second best result achieved in terms of accuracy was $90.4 \%$ by QSVM with 1000-feature subset, as shown in Table 14. The training time and prediction speed of proposed model on mixed views of PKU-Reid dataset are presented in Figure 16.

Table 14. Mixed view-based experimental results on PKU-Reid dataset.

\begin{tabular}{|c|c|c|c|c|c|c|c|c|c|c|c|c|}
\hline \multirow{2}{*}{$\begin{array}{l}\text { Classification } \\
\text { Methods }\end{array}$} & \multicolumn{5}{|c|}{ Optimized Feature Subsets } & \multicolumn{7}{|c|}{ Evaluation Metrics } \\
\hline & 100 & 250 & 500 & 750 & 1000 & $\mathrm{ACC}$ & AUC & SE & SP & PR & FM & GM \\
\hline LSVM & & & & & $\checkmark$ & 87.4 & 0.93 & 88.7 & 86.0 & 86.4 & 87.5 & 87.3 \\
\hline CSVM & & & & $\checkmark$ & & 91.2 & 0.96 & 91.4 & 91.0 & 91.0 & 91.2 & 91.2 \\
\hline CSVM & & & & & $\checkmark$ & 91.2 & 0.96 & 91.2 & 91.2 & 91.2 & 91.2 & 91.2 \\
\hline MGSVM & & & $\checkmark$ & & & 88.5 & 0.95 & 90.5 & 87.0 & 87.4 & 88.7 & 88.5 \\
\hline QSVM & & & & & $\checkmark$ & 90.4 & 0.95 & 91.1 & 89.8 & 89.9 & 90.5 & 90.4 \\
\hline FGSVM & $\checkmark$ & & & & & 56.7 & 0.59 & 97.9 & 15.5 & 53.7 & 69.4 & 39.0 \\
\hline FGSVM & & $\checkmark$ & & & & 56.7 & 0.59 & 97.9 & 15.5 & 53.7 & 69.4 & 39.0 \\
\hline CGSVM & & & & & $\checkmark$ & 87.1 & 0.91 & 89.1 & 85.1 & 85.7 & 87.3 & 87.1 \\
\hline FKNN & & & $\checkmark$ & & & 82.0 & 0.71 & 84.0 & 80.1 & 80.8 & 82.4 & 82.0 \\
\hline COKNN & & & & & $\checkmark$ & 81.8 & 0.87 & 89.7 & 73.9 & 77.4 & 83.1 & 81.4 \\
\hline CRKNN & & & & & $\checkmark$ & 79.1 & 0.87 & 86.5 & 71.7 & 75.4 & 80.6 & 78.8 \\
\hline
\end{tabular}

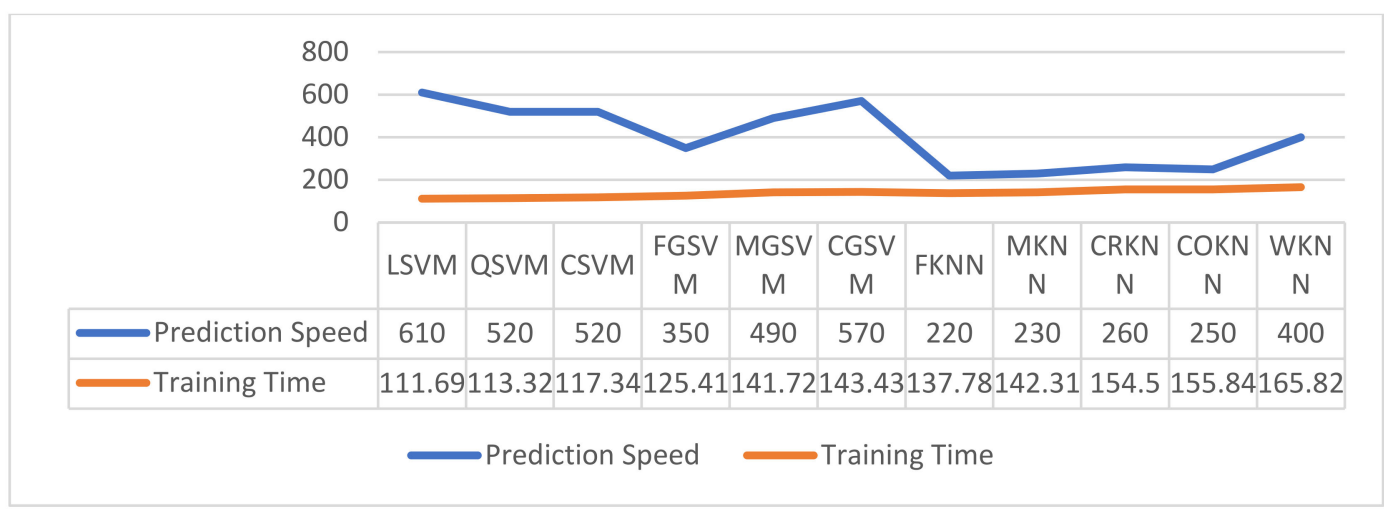

Figure 16. Proposed model training time (s) and prediction speed (obs/s) on mixed views of PKU-Reid dataset utilizing 1000-feature subset. 
The best ROC outcomes on the PKU-Reid dataset are presentedin Figure 17.

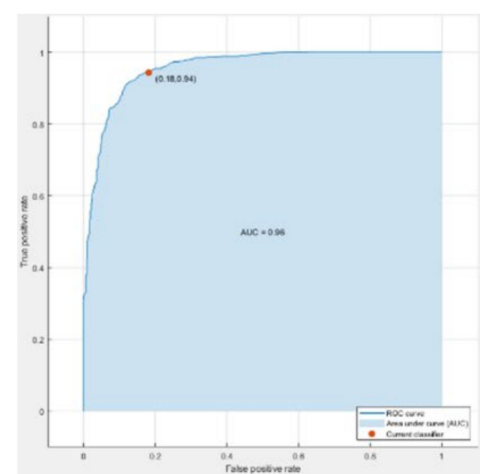

(a)

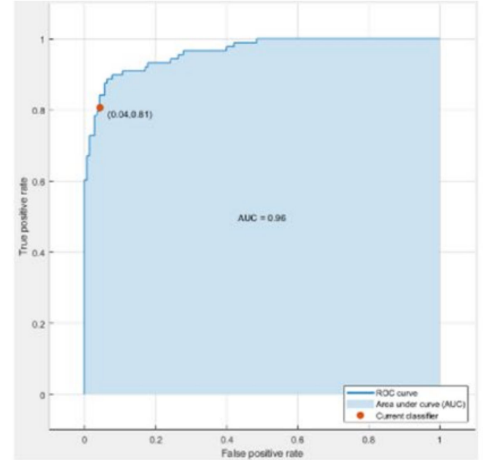

(b)

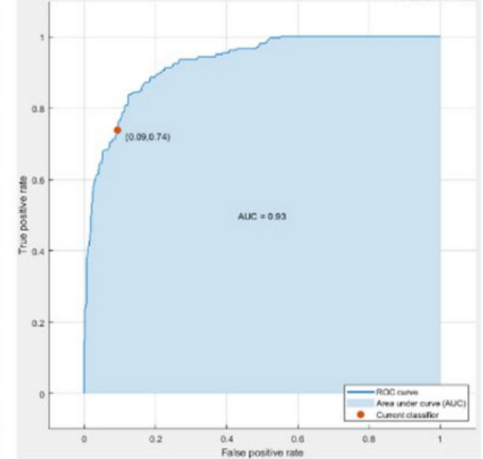

(c)

Figure 17. ROC curves and AUC values showing best outcomes of (a) mixed views with CSVM, (b) back views with CSVM, and (c) front views with CSVM of PKU-Reid dataset utilizing 1000-feature subset.

Regarding PKU-Reid dataset, it is pertinent to mention here that the relevant literature has been studied thoroughly to find existing methods in which PKU-Reid dataset is used and results are obtained for PGC purpose, but the literature lacks such methods; hence, a comparison of the results produced by the proposed approach is not possible. Although this dataset was introduced in 2016, researchers have not utilized it for PGC tasks.

\subsection{Performance Comparison between Proposed Approach and Existing Studies}

The proposed model was evaluated using frontal views, back views, and mixed views of MIT, VIPeR, and PKU-Reid testing datasets, and details of the results obtained are presented from Tables 5-14 in the previous section. A performance comparison between the proposed and existing classical and state-of-the-art methods is shown in the upcoming text. The results produced were compared with various methods such as CNN [16], HOG [45], HOG -LBP-HSV [45], CNN-e [19], Full-Body (CNN) [20], HDFL [40], SSAE [51], and recent best performers such as J-LDFR [41] and CSVFL [39] used in PGC for validation of the proposed framework.

These methods were selected for comparison because they have produced results in terms of accuracies on MIT dataset. Table 15 shows the comparison of accuracies achieved by existing pedestrian recognition methods and the proposed approach on MIT dataset. The highest accuracy, i.e., $85.4 \%$, obtained by the proposed framework on MIT dataset was generated by FKNN variant of KNN classifier. It can be observed from Table 15 that the proposed approach shows better accuracy; hence, it outperformed all the existing PGC methods. It accomplished $0.2 \%$ better ACC than the latest existing method, CSVFL [39], and $3.3 \%$ and $2.9 \%$ improvements as compared to the recent best performers J-LDFR [41] and SSAE [51], respectively. By comparing 74.3\% accuracy produced by HDFL [40] method with the proposed approach, it can be observed that the proposed method achieved $11.0 \%$ higher accuracy. A comparison of the results obtained with the existing and proposed methods in terms of accuracy is shown in Figure 18. 
Table 15. Performance comparison of results of proposed and existing PGC methods on MIT dataset.

\begin{tabular}{ccc}
\hline Methods & Year & ACC (\%) Using Mixed Views \\
\hline CNN [16] & 2013 & 80.4 \\
\hline HOG [45] & 2015 & 78.9 \\
\hline LBP [45] & 2015 & 76.1 \\
\hline HSV [45] & 2015 & 71.3 \\
\hline LBP-HSV [45] & 2015 & 77.6 \\
\hline HOG -HSV [45] & 2015 & 80.9 \\
\hline HOG -LBP [45] & 2015 & 79.8 \\
\hline HOG -LBP-HSV [45] & 2015 & 80.1 \\
\hline CNN-e [19] & 2017 & 81.5 \\
\hline Full-Body (CNN) [20] & 2017 & 82.0 \\
\hline HDFL [40] & 2018 & 74.3 \\
\hline SSAE [51] & 2018 & 82.4 \\
\hline J-LDFR [41] & 2021 & 82.0 \\
\hline CSVFL [39] & 2021 & 85.2 \\
\hline Proposed 4-BSMAB & Proposed & 85.4 \\
\hline
\end{tabular}

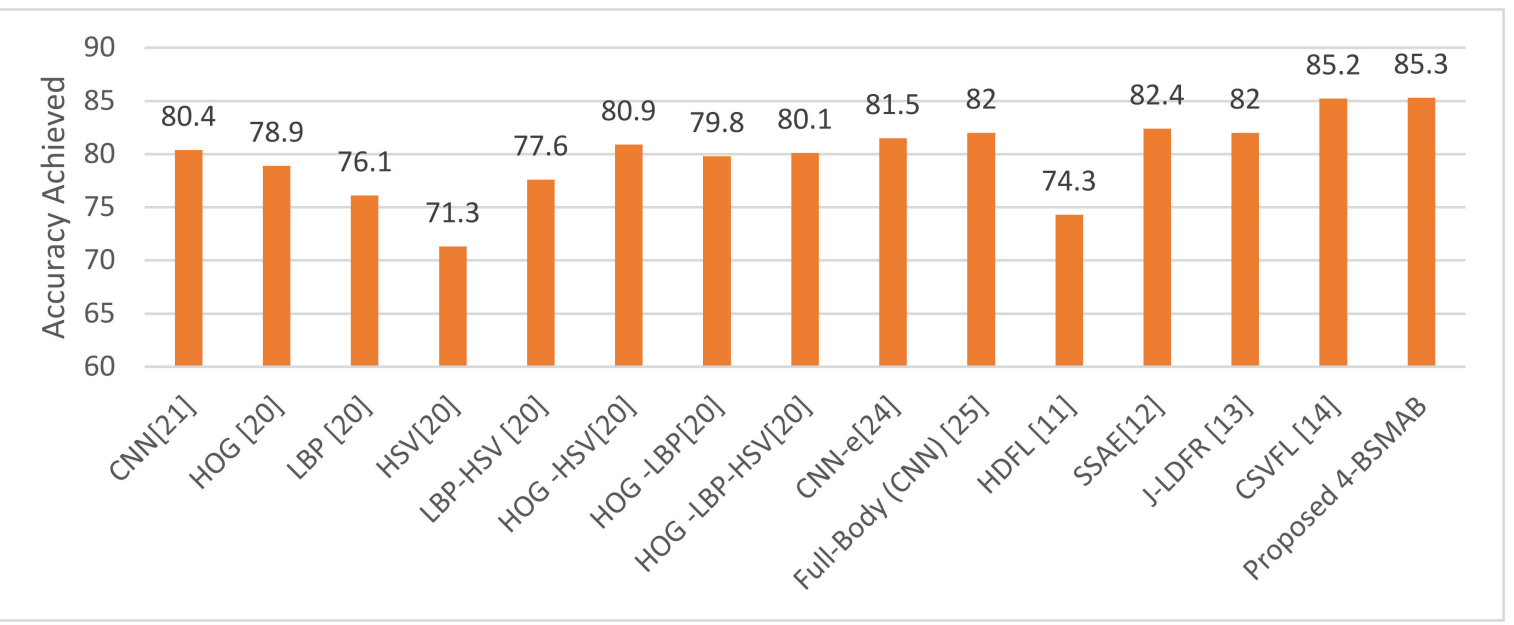

Figure 18. Comparison of accuracy obtained with proposed and existing approaches on MIT dataset.

To revalidate the worth of proposed method, the results obtained with the presented approach utilizing AUC evaluation protocol were also compared with existing methods. As per findings from relevant literature, J-LDFR [41] is the only technique that has computed an AUC on mixed views of MIT dataset. Table 16 shows the comparison in terms of AUC, and obtained results show that the proposed approach outperformed the existing method, J-LDFR [41], with a $6.0 \%$ improvement.

Table 16. AUC performance comparison between proposed and existing PGC approach on MIT dataset.

\begin{tabular}{ccc}
\hline Methods & Year & AUC (\%) Using Mixed Views \\
\hline J-LDFR [41] & 2021 & 86.0 \\
\hline Proposed 4-BSMAB & Proposed & 92.0 \\
\hline
\end{tabular}


Figure 19 shows AUC obtained by the proposed method using various classifiers with 1000-feature subset on mixed views of MIT dataset, and it can be observed that CSVM variant of SVM classifier produced highest AUC of $92.0 \%$ on mixed views of MIT dataset.

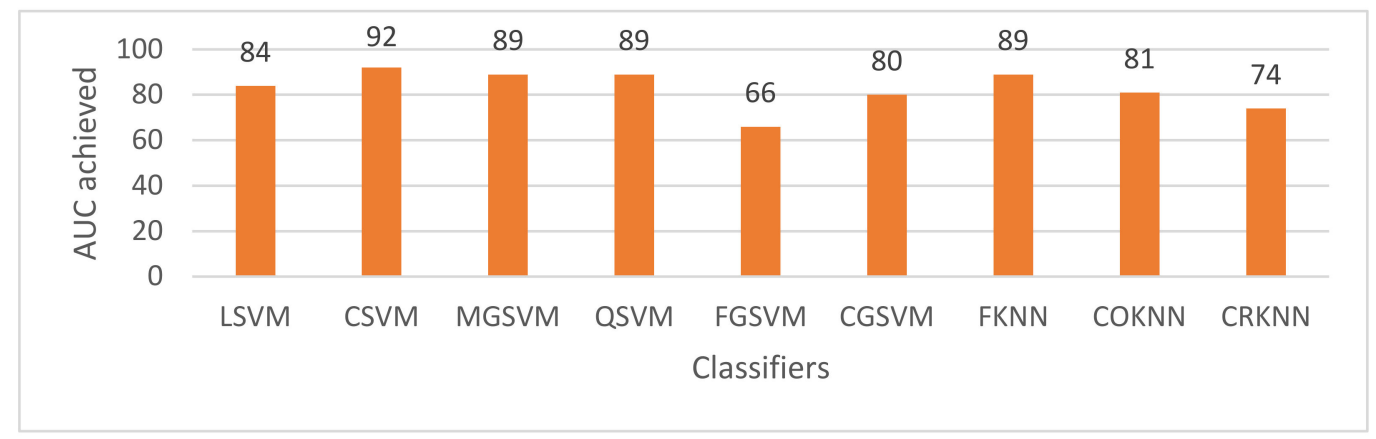

Figure 19. Comparison in terms of AUC with various classifiers on mixed views of MIT dataset using 1000-feature subset.

\subsection{Discussion}

In this manuscript, PGC problem was addressed, and for this purpose, pedestrian attribute recognition datasets such as MIT, VIPeR, and PKU-Reid were tested. Extensive experiments were performed to develop the proposed approach named as 4-BSMAB, having 64 layers for increased performance. First, CIFAR dataset of 100 classes was used for the training of the proposed model, and then features were extracted from three datasets such as MIT, VIPeR, and PKU-Reid using a pre-trained network. A feature optimization scheme based on ACS was selected to optimize the obtained features. The classification was carried out by performing experiments and selecting various optimal feature subsets, and the outcome of proposed framework was noted with the help of performance evaluation metrics.

At the time of feature selection, different variations of features were defined, and results were obtained applying these variations. The same classifiers were used in all experiments performed. Keeping in view the results obtained with the same classifiers on three different datasets, it was observed that the performance of most utilized classifiers increased as the number of optimized features in feature subsets increased, but, at the same time, the difference in accuracies provided by the classifiers became very small. On the other hand, it was also found that the performance of some classifiers decreased after the first iteration of 100 features and remained the same or increased at a very low rate between second and fourth iterations with feature subsets of 250 to 750, but in the fifth iteration with 1000 features, the performance increased at a very low rate. It was also noted that FKNN, CSVM, QSVM, and sometimes LSVM performed better than the other variants of KNN and SVM, whereas CRKNN, FGSVM, and CGSVM variants showed poor performances in most of the experiments, with a minimum accuracy of nearly $50.0 \%$. The experiments also showed that the performance of most classifiers is better when 500-, 750-, and 1000-feature subsets are used. Overall, 1000-feature subset can be considered best feature subset. It was also seen that the performance of all variants of KNN related to the training time and prediction speed was found to be higher in comparison with SVM.

\section{Conclusions}

A novel CNN-based framework, 4-BSMAB, was assessed for feature extraction, and ACS was used for the selection of optimized feature sets. The SoftMax classifier was utilized to train 4-BSMAB model on the existing CIFAR-100 dataset, and features were obtained from common pedestrian datasets. An optimized feature set obtained with ACS optimization technique was provided to various classifiers of SVM and KNN for PGC. Five-fold-type cross-validation was carried out to train and test the pedestrian datasets. Extensive experimentation was carried out with various feature subsets, and the details of only five experiments conducted on each dataset were mentioned. It was observed from the experimentation results that the optimized feature subset with 100 features 
produced a lower accuracy of $81.3 \%$, whereas 1000 -featuresubset performed better and achieved $85.4 \%$ accuracy with FKNN classifier, and 92\% AUC with CSVM classifier, on MIT dataset. A comparison of the results of proposed model and existing state-of-the-art methods on MIT dataset was presented, and it was observed that the proposed method outperformed existing gender classification approaches. It was also noted that CSVM classifier performed better on PKU-Reid dataset and generated 93\% accuracy and 96\% AUC. The experimentation results also show that most of the classifiers produced better results with 1000-optimized feature subset and obtained second best results with an optimized feature subset of 100 features. As per findings, results on PKU-Reid are not available in the relevant literature, and a performance comparison in this regard is not possible. Although the proposed framework produced satisfactory results, the accuracy can still be improved further. In future work, other approaches such as LSTMs, manifold learning, and quantum deep learning may be explored for better performance.

Author Contributions: Conceptualization, F.A., M.Y. and M.F.; Data curation, F.A., M.Y. and M.F.; Formal analysis, F.A., M.Y., M.F., M.A.E., S.L. and A.A.A.E.-L.; Funding acquisition, M.A.E., S.L. and A.A.A.E.-L.; Investigation, F.A., M.Y. and M.F.; Methodology, F.A., M.Y. and M.F.; Writing-review \& editing, F.A., M.Y., M.F., M.A.E., S.L. and A.A.A.E.-L. All authors have read and agreed to the published version of the manuscript.

Funding: This work is supported by the Hubei Provincial Science and Technology Major Project of China under Grant No. 2020AEA011 and the Key Research \& Development Plan of Hubei Province of China under Grant No. 2020BAB100 and the project of Science, Technology and Innovation Commission of Shenzhen Municipality of China under Grant No. JCYJ20210324120002006.

Data Availability Statement: The dataset download link with annotations is available at: http: //mmlab.ie.cuhk.edu.hk/projects / PETA.html and https:/ / paperswithcode.com/dataset/peta.

Conflicts of Interest: The authors declare no conflict of interest.

\section{References}

1. Sivabalakrishnan, M.; Menaka, R.; Jeeva, S. Smart video surveillance systems and identification of human behavior analysis. In Countering Cyber Attacks and Preserving the Integrity and Availability of Critical Systems; IGI Global: Philadelphia, PA, USA, 2019; pp. 64-97.

2. Filonenko, A.; Jo, K.-H. Unattended object identification for intelligent surveillance systems using sequence of dual background difference. IEEE Trans. Ind. Inform. 2016, 12, 2247-2255.

3. Zhu, H.; Wei, H.; Li, B.; Yuan, X.; Kehtarnavaz, N. A Review of Video Object Detection: Datasets, Metrics and Methods. Appl. Sci. 2020, 10, 7834. [CrossRef]

4. Jang, D.-H.; Kwon, K.-S.; Kim, J.-K.; Yang, K.-Y.; Kim, J.-B. Dog Identification Method Based on Muzzle Pattern Image. Appl. Sci. 2020, 10, 8994. [CrossRef]

5. Rybak, Ł.; Dudczyk, J. A geometrical divide of data particle in gravitational classification of moons and circles data sets. Entropy 2020, 22, 1088. [CrossRef]

6. Rybak, Ł.; Dudczyk, J. Variant of Data Particle Geometrical Divide for Imbalanced Data Sets Classification by the Example of Occupancy Detection. Appl. Sci. 2021, 11, 4970. [CrossRef]

7. Feng, Q.; Yuan, C.; Pan, J.-S.; Yang, J.-F.; Chou, Y.-T.; Zhou, Y.; Li, W. Superimposed sparse parameter classifiers for face recognition. IEEE Trans. Cybern. 2016, 47, 378-390. [CrossRef]

8. Neff, C.; Mendieta, M.; Mohan, S.; Baharani, M.; Rogers, S.; Tabkhi, H. REVAMP 2 T: Real-Time Edge Video Analytics for Multicamera Privacy-Aware Pedestrian Tracking. IEEE Internet Things J. 2019, 7, 2591-2602. [CrossRef]

9. Xiao, T.; Li, H.; Ouyang, W.; Wang, X. Learning deep feature representations with domain guided dropout for person reidentification. In Proceedings of the IEEE Conference on Computer Vision and Pattern Recognition, Las Vegas, NV, USA, 27-30 June 2016; pp. 1249-1258.

10. Wu, S.; Chen, Y.-C.; Li, X.; Wu, A.-C.; You, J.-J.; Zheng, W.-S. An enhanced deep feature representation for person re-identification. In Proceedings of the 2016 IEEE Winter Conference on Applications of Computer Vision (WACV 2016), Lake Placid, NY, USA, 7-10 March 2016; IEEE: Piscataway, NJ, USA, 2016; pp. 1-8.

11. Yao, H.; Zhang, S.; Hong, R.; Zhang, Y.; Xu, C.; Tian, Q. Deep representation learning with part loss for person re-identification. IEEE Trans. Image Process. 2019, 28, 2860-2871. [CrossRef]

12. Kong, Y.; Ding, Z.; Li, J.; Fu, Y. Deeply learned view-invariant features for cross-view action recognition. IEEE Trans. Image Process. 2017, 26, 3028-3037. [CrossRef] 
13. Khan, M.A.; Akram, T.; Sharif, M.; Javed, M.Y.; Muhammad, N.; Yasmin, M. An implementation of optimized framework for action classification using multilayers neural network on selected fused features. Pattern Anal. Appl. 2019, 22, $1377-1397$. [CrossRef]

14. Ng, C.B.; Tay, Y.H.; Goi, B.-M. Recognizing human gender in computer vision: A survey. In Pacific Rim International Conference on Artificial Intelligence, Kuching, Malaysia, 3-7 September 2012; Springer: Berlin/Heidelberg, Germany, 2012; pp. 335-346.

15. Bhatnagar, G.; Wu, Q.J. A fractal dimension based framework for night vision fusion. IEEE/CAA J. Autom. Sin. 2018, 6, 220-227. [CrossRef]

16. $\mathrm{Ng}$, C.-B.; Tay, Y.-H.; Goi, B.-M. A convolutional neural network for pedestrian gender recognition. In International Symposium on Neural Networks, Dalian, China, 4-6 July 2013; Springer: Berlin/Heidelberg, Germany, 2013; pp. 558-564.

17. Antipov, G.; Berrani, S.-A.; Ruchaud, N.; Dugelay, J.-L. Learned vs. hand-crafted features for pedestrian gender recognition. In Proceedings of the 23rd ACM International Conference on Multimedia, Brisbane, Australia, 26-30 October 2015; ACM: New York, NY, USA, 2015; pp. 1263-1266.

18. Ng, C.-B.; Tay, Y.-H.; Goi, B.-M. Comparing image representations for training a convolutional neural network to classify gender. In Proceedings of the 1st International Conference on Artificial Intelligence, Modelling and Simulation, Kota Kinabalu, Malaysia, 3-5 December 2013; IEEE: Piscataway, NJ, USA, 2013; pp. 29-33.

19. Ng, C.-B.; Tay, Y.-H.; Goi, B.-M. Training strategy for convolutional neural networks in pedestrian gender classification. In Second International Workshop on Pattern Recognition; International Society for Optics and Photonics: Bellingham, WA, USA, 2017; Volume 10443, p. 104431A.

20. Raza, M.; Zonghai, C.; Rehman, S.U.; Zhenhua, G.; Jikai, W.; Peng, B. Part-wise pedestrian gender recognition via deep convolutional neural networks. In Proceedings of the 2nd IET International Conference on Biomedical Image and Signal Processing (ICBISP 2017), Wuhan, China, 13-14 May 2017.

21. Sun, Y.; Zhang, M.; Sun, Z.; Tan, T. Demographic analysis from biometric data: Achievements, challenges, and new frontiers. IEEE Trans. Pattern Anal. Mach. Intell. 2017, 40, 332-351. [CrossRef]

22. Azzopardi, G.; Greco, A.; Saggese, A.; Vento, M. Fusion of domain-specific and trainable features for gender recognition from face images. IEEE Access 2018, 6, 24171-24183. [CrossRef]

23. Mane, S.; Shah, G. Facial recognition, expression recognition, and gender identification. In Data Management, Analytics and Innovation; Springer: Berlin/Heidelberg, Germany, 2019; pp. 275-290.

24. Cheng, J.; Li, Y.; Wang, J.; Yu, L.; Wang, S. Exploiting effective facial patches for robust gender recognition. Tsinghua Sci. Technol. 2019, 24, 333-345. [CrossRef]

25. Geetha, A.; Sundaram, M.; Vijayakumari, B. Gender classification from face images by mixing the classifier outcome of prime, distinct descriptors. Soft Comput. 2019, 23, 2525-2535. [CrossRef]

26. Tapia, J.E.; Perez, C.A. Gender classification based on fusion of different spatial scale features selected by mutual information from histogram of LBP, intensity, and shape. IEEE Trans. Inf. Forensics Secur. 2013, 8, 488-499. [CrossRef]

27. Tapia, J.E.; Perez, C.A.; Bowyer, K.W. Gender classification from the same iris code used for recognition. IEEE Trans. Inf. Forensics Secur. 2016, 11, 1760-1770. [CrossRef]

28. Shan, C. Learning local binary patterns for gender classification on real-world face images. Pattern Recognit. Lett. 2012, 33, 431-437. [CrossRef]

29. Lemley, J.; Bazrafkan, S.; Corcoran, P. Deep Learning for Consumer Devices and Services: Pushing the limits for machine learning, artificial intelligence, and computer vision. IEEE Consum. Electron. Mag. 2017, 6, 48-56. [CrossRef]

30. Ahmad, K.; Sohail, A.; Conci, N.; de Natale, F. A comparative study of global and deep features for the analysis of user-generated natural disaster related images. In Proceedings of the IEEE 13th Image, Video, and Multidimensional Signal Processing Workshop (IVMSP), Aristi Village, Greece, 10-12 June 2018; IEEE: Piscataway, NJ, USA, 2018; pp. 1-5.

31. Deng, W.; Zheng, L.; Ye, Q.; Kang, G.; Yang, Y.; Jiao, J. Image-image domain adaptation with preserved self-similarity and domain-dissimilarity for person re-identification. In Proceedings of the IEEE Conference on Computer Vision and Pattern Recognition, Salt Lake City, UT, USA, 18-23 June 2018; pp. 994-1003.

32. Tang, Y.; Yang, X.; Wang, N.; Song, B.; Gao, X. CGAN-TM: A novel domain-to-domain transferring method for person reidentification. IEEE Trans. Image Process. 2020, 29, 5641-5651. [CrossRef]

33. Wei, L.; Zhang, S.; Gao, W.; Tian, Q. Person transfer gan to bridge domain gap for person re-identification. In Proceedings of the IEEE Conference on Computer Vision and Pattern Recognition, Salt Lake City, UT, USA, 18-23 June 2018; pp. 79-88.

34. Ren, C.-X.; Liang, B.; Ge, P.; Zhai, Y.; Lei, Z. Domain adaptive person re-identification via camera style generation and label propagation. IEEE Trans. Inf. Forensics Secur. 2019, 15, 1290-1302. [CrossRef]

35. Karanam, S.; Li, Y.; Radke, R.J. Person re-identification with discriminatively trained viewpoint invariant dictionaries. In Proceedings of the IEEE International Conference on Computer Vision, Santiago, Chile, 7-13 December 2015; pp. 4516-4524.

36. Li, S.; Shao, M.; Fu, Y. Person re-identification by cross-view multi-level dictionary learning. IEEE Trans. Pattern Anal. Mach. Intell. 2017, 40, 2963-2977. [CrossRef] [PubMed]

37. Xu, D.; Chen, J.; Liang, C.; Wang, Z.; Hu, R. Cross-view identical part area alignment for person re-identification. In Proceedings of the ICASSP 2019-2019 IEEE International Conference on Acoustics, Speech and Signal Processing (ICASSP), Brighton, UK, 12-17 May 2019; pp. 2462-2466. 
38. Chen, Y.-C.; Zhu, X.; Zheng, W.-S.; Lai, J.-H. Person re-identification by camera correlation aware feature augmentation. IEEE Trans. Pattern Anal. Mach. Intell. 2017, 40, 392-408. [CrossRef]

39. Cai, L.; Zeng, H.; Zhu, J.; Cao, J.; Wang, Y.; Ma, K.-K. Cascading Scene and Viewpoint Feature Learning for Pedestrian Gender Recognition. IEEE Internet Things J. 2020, 8, 3014-3026. [CrossRef]

40. Cai, L.; Zhu, J.; Zeng, H.; Chen, J.; Cai, C.; Ma, K.-K. Hog-assisted deep feature learning for pedestrian gender recognition. J. Frankl. Inst. 2018, 355, 1991-2008. [CrossRef]

41. Fayyaz, M.; Yasmin, M.; Sharif, M.; Raza, M. J-LDFR: Joint low-level and deep neural network feature representations for pedestrian gender classification. Neural Comput. Appl. 2021, 33, 361-391. [CrossRef]

42. Cao, L.; Dikmen, M.; Fu, Y.; Huang, T.S. Gender recognition from body. In Proceedings of the 16th ACM International Conference on Multimedia, Vancouver, BC, Canada, 26-31 October 2008; ACM: New York, NY, USA, 2008; pp. 725-728.

43. Guo, G.; Mu, G.; Fu, Y. Gender from body: A biologically-inspired approach with manifold learning. In Proceedings of the Asian Conference on Computer Vision, Xi'an, China, 23-27 September 2009; Springer: Berlin/Heidelberg, Germany, 2009 ; pp. 236-245.

44. Collins, M.; Zhang, J.; Miller, P.; Wang, H. Full body image feature representations for gender profiling. In Proceedings of the 2009 IEEE 12th International Conference on Computer Vision Workshops, ICCV Workshops, Kyoto, Japan, 27 September-4 October 2009; pp. 1235-1242.

45. Geelen, C.D.; Wijnhoven, R.G.; Dubbelman, G. Gender classification in low-resolution surveillance video: In-depth comparison of random forests and SVMs. In Video Surveillance and Transportation Imaging Applications; International Society for Optics and Photonics: Bellingham, WA, USA, 2015; Volume 9407, p. 94070M.

46. Sindagi, V.A.; Patel, V.M. A survey of recent advances in cnn-based single image crowd counting and density estimation. Pattern Recognit. Lett. 2018, 107, 3-16. [CrossRef]

47. Li, C.; Guo, J.; Porikli, F.; Pang, Y. Lightennet: A convolutional neural network for weakly illuminated image enhancement. Pattern Recognit. Lett. 2018, 104, 15-22. [CrossRef]

48. Rashid, M.; Khan, M.A.; Sharif, M.; Raza, M.; Sarfraz, M.M.; Afza, F. Object detection and classification: A joint selection and fusion strategy of deep convolutional neural network and SIFT point features. Multimed. Tools Appl. 2019, 78, 15751-15777. [CrossRef]

49. Khan, M.A.; Akram, T.; Sharif, M.; Awais, M.; Javed, K.; Ali, H.; Saba, T. CCDF: Automatic system for segmentation and recognition of fruit crops diseases based on correlation coefficient and deep CNN features. Comput. Electron. Agric. 2018, 155, 220-236. [CrossRef]

50. Sharif, M.; Khan, M.A.; Rashid, M.; Yasmin, M.; Afza, F.; Tanik, U.J. Deep CNN and geometric features-based gastrointestinal tract diseases detection and classification from wireless capsule endoscopy images. J. Exp. Theor. Artif. Intell. 2019, 33, 1-23. [CrossRef]

51. Raza, M.; Sharif, M.; Yasmin, M.; Khan, M.A.; Saba, T.; Fernandes, S.L. Appearance based pedestrians' gender recognition by employing stacked auto encoders in deep learning. Future Gener. Comput. Syst. 2018, 88, 28-39. [CrossRef]

52. Cai, L.; Zhu, J.; Zeng, H.; Chen, J.; Cai, C. Deep-learned and hand-crafted features fusion network for pedestrian gender recognition. In Proceedings of the ELM-2016, Singapore, 13-15 December 2016; Springer: Berlin/Heidelberg, Germany, 2018 ; pp. $207-215$.

53. Ng, C.-B.; Tay, Y.-H.; Goi, B.-M. Pedestrian gender classification using combined global and local parts-based convolutional neural networks. Pattern Anal. Appl. 2018, 22, 1469-1480. [CrossRef]

54. Krizhevsky, A.; Sutskever, I.; Hinton, G.E. Imagenet classification with deep convolutional neural networks. Adv. Neural Inf. Process. Syst. 2012, 25, 1097-1105. [CrossRef]

55. Balocco, S.; González, M.; Nanculef, R.; Radeva, P.; Thomas, G. Calcified plaque detection in IVUS sequences: Preliminary results using convolutional nets. In Proceedings of the International Workshop on Artificial Intelligence and Pattern Recognition, Havana, Cuba, 24-26 September 2018; Springer: Berlin/Heidelberg, Germany, 2018; pp. 34-42.

56. Ioffe, S.; Szegedy, C. Batch normalization: Accelerating deep network training by reducing internal covariate shift. In Proceedings of the International Conference on Machine Learning, Lille, France, 6-11 July 2015; pp. 448-456.

57. Liu, Y.; Wang, X.; Wang, L.; Liu, D. A modified leaky ReLU scheme (MLRS) for topology optimization with multiple materials. Appl. Math. Comput. 2019, 352, 188-204. [CrossRef]

58. Bouvrie, J. Notes on convolutional neural networks; MIT CBCL Technical Report. Neural Nets 2006, 5869, 47-60.

59. Li, Y.; Hao, Z.; Lei, H. Survey of convolutional neural network. J. Comput. Appl. 2016, 36, 2508-2515.

60. Wu, J. Introduction to convolutional neural networks. In National Key Lab for Novel Software Technology; Nanjing University: Nanjing, China, 2017; Volume 5, p. 23.

61. Krizhevsky, A.; Hinton, G. Learning Multiple Layers of Features From Tiny Images; Technical Report; University of Toronto: Toronto, ON, Canada, 2009.

62. Dash, M.; Liu, H. Feature selection for clustering. In Pacific-Asia Conference on Knowledge Discovery and Data Mining; Springer: Berlin/Heidelberg, Germany, 2000; pp. 110-121.

63. Rashno, A.; Nazari, B.; Sadri, S.; Saraee, M. Effective pixel classification of mars images based on ant colony optimization feature selection and extreme learning machine. Neurocomputing 2017, 226, 66-79. [CrossRef]

64. Noble, W.S. What is a support vector machine? Nat. Biotechnol. 2006, 24, 1565-1567. [CrossRef]

65. Peterson, L.E. K-nearest neighbor. Scholarpedia 2009, 4, 1883. [CrossRef]

66. Chang, Y.-W.; Lin, C.-J. Feature ranking using linear SVM. In Proceedings of the Workshop on the Causation and Prediction Challenge at WCCI 2008, Hong Kong, China, 3-4 June 2008; pp. 53-64.

67. Dagher, I. Quadratic kernel-free non-linear support vector machine. J. Glob. Optim. 2008, 41, 15-30. [CrossRef] 
68. Virdi, P.; Narayan, Y.; Kumari, P.; Mathew, L. Discrete wavelet packet based elbow movement classification using fine Gaussian SVM. In Proceedings of the IEEE 1st International Conference on Power Electronics, Intelligent Control and Energy Systems (ICPEICES), Delhi, India, 4-6 July 2016; pp. 1-5.

69. Liu, Z.; Zuo, M.J.; Zhao, X.; Xu, H. An Analytical Approach to Fast Parameter Selection of Gaussian RBF Kernel for Support Vector Machine. J. Inf. Sci. Eng. 2015, 31, 691-710.

70. Rüping, S. SVM Kernels for Time Series Analysis; Technical Report; TU Dortmund: Dortmund, Germany, 2001; pp. 1-13. [CrossRef]

71. Ayat, N.-E.; Cheriet, M.; Suen, C.Y. Automatic model selection for the optimization of SVM kernels. Pattern Recognit. 2005, 38, 1733-1745. [CrossRef]

72. Haasdonk, B. Feature space interpretation of SVMs with indefinite kernels. IEEE Trans. Pattern Anal. Mach. Intell. 2005, 27, 482-492. [CrossRef]

73. Xu, Y.; Zhu, Q.; Fan, Z.; Qiu, M.; Chen, Y.; Liu, H. Coarse to fine K nearest neighbor classifier. Pattern Recognit. Lett. 2013, 34, 980-986. [CrossRef]

74. Singh, A.P. Analysis of variants of KNN algorithm based on preprocessing techniques. In Proceedings of the 2018 International Conference on Advances in Computing, Communication Control and Networking (ICACCCN), Greater Noida, India, 12-13 October 2018; pp. 186-191.

75. Lamba, A.; Kumar, D. Survey on KNN and its variants. Int. J. Adv. Res. Comput. Commun. Eng. 2016, 5, 430-435.

76. Jiang, L.; Zhang, H.; Su, J. Learning k-nearest neighbor naive bayes for ranking. In Proceedings of the International Conference on Advanced Data Mining and Applications, Guilin, China, 19-21 December 2005; Springer: Berlin/Heidelberg, Germany, 2005; pp. 175-185.

77. Gray, D.; Tao, H. Viewpoint invariant pedestrian recognition with an ensemble of localized features. In Proceedings of the Computer Vision-ECCV 2008, Marseille, France, 12-18 August 2008; ACM: New York, NY, USA, 2008; pp. $262-275$.

78. Deng, Y.; Luo, P.; Loy, C.C.; Tang, X. Pedestrian attribute recognition at far distance. In Proceedings of the 22nd ACM International Conference on Multimedia, Orlando, FL, USA, 3-7 November 2014; ACM: New York, NY, USA, 2014; pp. 789-792.

79. Ma, L.; Liu, H.; Hu, L.; Wang, C.; Sun, Q. Orientation driven bag of appearances for person re-identification. arXiv 2016, arXiv:1605.02464. 